\title{
Starting flow in channels with boundary slip
}

\author{
Chiu-On $\mathrm{Ng}^{1}$ \\ Department of Mechanical Engineering, The University of Hong Kong, \\ Pokfulam Road, Hong Kong
}

January 13, 2016

\begin{abstract}
This paper contains a collection of problems and their analytical solutions for starting flows under the effect of boundary slip in channels of various geometries. The startup flows examined in this work include: (i) plane Couette flow between two plates, (ii) rotary Couette flow between two coaxial cylinders, (iii) Poiseuille flow through a parallel-plate channel, (iv) Poiseuille flow through a rectangular channel, (v) Poiseuille flow through a circular channel, and (iv) Poiseuille flow through an annulus. It is first shown that, using a depletion layer to model the effective slip, the slip length may attain its steady state much faster than the starting flow will do. This supports the use of a constant Navier slip length in the present problems. Transient solutions are derived in the form of eigenfunction expansions, where the eigenvalues are determined, by solving the characteristic equations numerically, as a function of the channel geometry and the slip lengths. From the leading eigenvalue, it is found that the boundary slip will in general lengthen the transient period of a starting flow.
\end{abstract}

Keywords: Start-up flow; Navier slip condition; eigenfunction expansion.

\footnotetext{
${ }^{1}$ Tel: (852) 2859 2622; Fax: (852) 28585415 ; E-mail address: cong@hku.hk (C.-O. Ng).
} 


\section{Introduction}

There exists a sizable body of literature on flow through channels with boundary slip, where the slip may arise from rarefaction effect, superhydrophobicity, or intrinsic slippage. Most of the studies are, however, for steady flows, and unsteady slip flows have not received much attention until recently.

An early study on transient slip flow was due to Bestman et al. [1], who revisited the classical Rayleigh problem of flow driven by the impulsive motion of a flat plate, on using a slip, instead of the standard no-slip, boundary condition. de Socio and Marino [2] took it further to investigate unsteady compressible flow and heat transfer over an impulsively started plate subjected to velocity slip and temperature jump boundary conditions. Transient slip flow and heat transfer in microchannels was then studied by Castellões et al. [3]. They presented hybrid analytical-numerical solutions for time-dependent slip flow in parallel-plate or circular channel, where the transient motion is caused by flow start-up with a step change, or a periodic time variation of the pressure gradient. Unsteady slip flows forced by a time-periodic pressure gradient through circular, annular and rectangular microchannels were investigated by $\mathrm{Wu}$ et al. [4], and Wiwatanapataphee et al. [5,6]. More recently, Crane and McVeigh [7] studied unsteady axisymmetric slip flow along an impulsively started cylinder.

In view of the growing interest in impulsively started slip flows, it is our objective here to look into the start-up (i.e., motion from rest) of some internal flows subject to boundary slip for a variety of channel geometries. The start-up flow may arise from a sudden movement of a boundary at constant velocity in its own plane, known as starting Couette flow, or a suddenly imposed constant pressure gradient, known as starting Poiseuille flow. We shall present in this paper the problems as well as their analytical solutions for starting Couette flow between two plates or between two coaxial cylinders, and starting Poiseuille flow through a parallelplate, rectangular, circular, or annular channel, all subject to the condition of boundary slip. Unlike external flows, these transient internal flows will asymptotically tend to a steady state at large times. We shall derive the exponentially decaying transient solutions using the eigenfunction expansion method, as has been applied by Wang [8-10].

Most of the solutions presented in this paper have not been reported before, or are more 
general than those existing in the literature. A few problems have been studied before, but are included here for completeness of this paper. Start-up pressure-driven flows with slip in parallel-plate, circular and rectangular channels were examined recently by Avramenko et al. [11,12], who solved the problems by Laplace transform or series solution methods. Our solutions are expressed in a form simpler than theirs. Matthews and Hastie [13] also studied, using Fourier series expansions, start-up pressure-driven flow through a parallel-plate channel with disparate slip lengths on the two plates. We may check our deduction by comparing with their solution for this problem. Using a dynamic slip model to account for slip relaxation, Kaoullas and Georgiou [14] presented analytical solutions for starting and ceasing Poiseuille and Couette flows in circular and parallel-plate channels. We may also check our solutions, in the case of equal slip lengths on the walls, against those by Kaoullas and Georgiou [14] without the dynamic slip.

As was remarked by Matthews and Hastie [13] and Avramenko et al. [11,12], these analytical solutions are valuable for future studies. For one thing, they can be used as benchmarks for numerical simulations, or to validate computational fluid dynamics (CFD) codes. For another, further studies, such as heat transfer, rheological measurement, flow control optimization or flow instability, may be pursued based on the analytical solutions provided here.

To model the boundary slip, we shall adopt the Navier slip condition, which states that the slip velocity is linearly proportional to the near-wall shear stress. For Newtonian fluids, the proportionality constant multiplied by the fluid viscosity is known as the slip length. The slip length, which measures the depth into the wall where the velocity profile would extrapolate to zero, is the core parameter in the Navier slip condition. While the slip condition works well for steady flow, the application of this condition to unsteady flow is subject to scrutiny. Wang [15] found that in time-oscillatory flow the slip velocity and the local shear stress are in general out of phase, and consequently the ratio of these quantities varies periodically with time. Ng and Wang [16] further deduced that, for oscillatory flow through a channel bounded by patterned walls, the effective slip length is a complex quantity in order to account for the phase shift between the velocity and velocity gradient.

A central question needs to be answered before we may apply the Navier slip condition to 
the present problems: can the slip length be treated as a steady quantity in starting flows? To answer this question, we first consider in Section 2 starting flow in a coated channel, where the slip at a wall comes to effect through the action of a low-viscosity depletion layer near the wall; see Fig. 1. We shall show that as long as the depletion layer is much thinner than the bulk fluid layer, the transient of the slip length will decay much faster than that of the starting flow itself. In other words, the slip length will attain its steady-state value much more quickly than the flow will do. On assuming steady and uniform slip lengths, our problems for starting Couette flow between two parallel flat plates and between two coaxial cylinders, and starting pressure-driven flow through a parallel-plate, rectangular, circular, and annular channel are then presented in Sections 3-8. Figures 1, 3, 6, 8, 10, 13, 16 show the definition sketches for these problems.

One-dimensional laminar Newtonian fluid flows are assumed. The problems are solved by the classical method of separation of variables, and the solutions come in the form of Fourier series expansions. As the solution method is rather standard, we shall leave out most of the mathematical details here. The focus is on the key equations, including the problem specific characteristic equations that determine the eigenvalues. For ease of reference by other researchers, we shall perform the deduction with physical quantities, and express the deduced equations in both dimensional and non-dimensional forms. Solutions for the no-slip case will be checked against those known in the literature, if any.

\section{Couette flow between two plates with a depletion layer}

We first consider starting Couette flow between two parallel flat plates, where the boundary slip comes into effect through the action of a near-wall depletion layer. This problem aims to reveal that the transient duration of the boundary slip is typically much shorter than that of the starting flow itself.

As depicted in Fig. 1, the problem is to examine the start-up flow of a bulk fluid that is lubricated by a thin depletion layer of thickness $a$ near the upper plate, while driven by the 


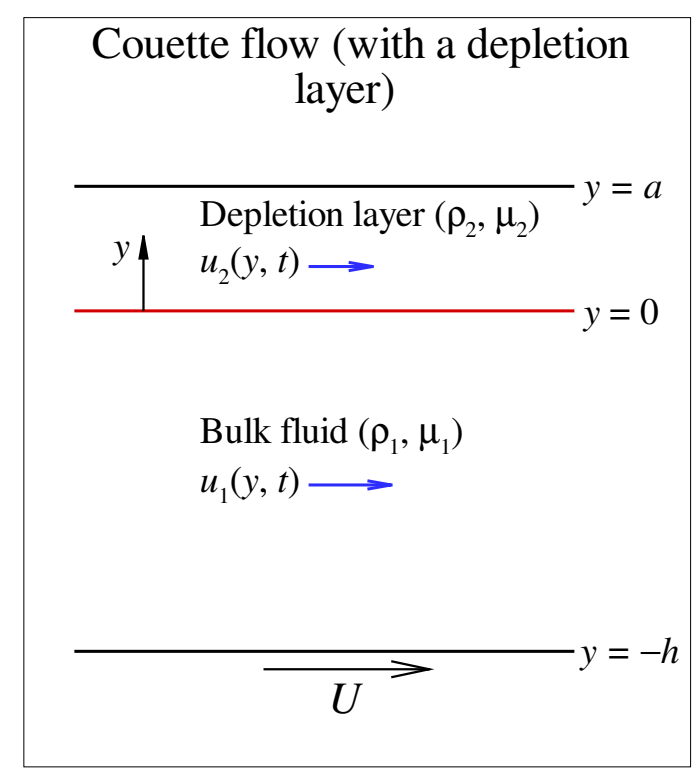

Figure 1: Starting Couette flow between two parallel flat plates (effective slip through the action of a thin depletion layer near the upper plate).

sudden motion in its own plane of the lower plate. The depletion layer is by definition a layer occupied by a low-density and low-viscosity fluid. Hence, the density ratio $\rho_{1} / \rho_{2}$ and the dynamic viscosity ratio $\mu_{1} / \mu_{2}$ are both much larger than unity, where the subscripts 1 and 2 denote the bulk fluid and the depletion-layer-fluid, respectively. On the other hand, the depletion layer is typically much thinner than the bulk fluid layer, and hence the thickness ratio $a / h$ is a very small number, where $h$ is the thickness of the bulk fluid layer.

Flow of the two fluids is governed by:

$$
\begin{gathered}
\frac{\partial u_{1}}{\partial t}=\nu_{1} \frac{\partial^{2} u_{1}}{\partial y^{2}} \quad \text { in }-h \leq y<0 \\
\frac{\partial u_{2}}{\partial t}=\nu_{2} \frac{\partial^{2} u_{2}}{\partial y^{2}} \quad \text { in } 0<y \leq a
\end{gathered}
$$

where $u(y, t)$ is the fluid velocity, and $\nu=\mu / \rho$ is the kinematic viscosity. The boundary conditions are

$$
\begin{gathered}
u_{1}=\left\{\begin{array}{l}
U, \quad t>0 \\
0, \quad t<0
\end{array} \quad \text { at } y=-h,\right. \\
u_{1}=u_{2}, \quad \mu_{1} \frac{\partial u_{1}}{\partial y}=\mu_{2} \frac{\partial u_{2}}{\partial y} \quad \text { at } y=0,
\end{gathered}
$$




$$
u_{2}=0 \quad \text { at } y=a,
$$

where $U$ is a positive constant. The solution to this problem is obtainable from Carslaw and Jaeger [17]:

$$
\begin{aligned}
& u_{1}(y, t)=\frac{U(\alpha a-y)}{\alpha a+h}-2 U \sum_{n=1}^{\infty} \frac{\sin ^{2}\left(k \beta_{n} a\right) \sin \left[\beta_{n}(h+y)\right]}{\beta_{n}\left[h \sin ^{2}\left(k \beta_{n} a\right)+\sigma k a \sin ^{2}\left(\beta_{n} h\right)\right]} e^{-\nu_{1} \beta_{n}^{2} t}, \\
& u_{2}(y, t)=\frac{\alpha U(a-y)}{\alpha a+h}-2 U \sum_{n=1}^{\infty} \frac{\sin \left(\beta_{n} h\right) \sin \left(k \beta_{n} a\right) \sin \left[k \beta_{n}(a-y)\right]}{\beta_{n}\left[h \sin ^{2}\left(k \beta_{n} a\right)+\sigma k a \sin ^{2}\left(\beta_{n} h\right)\right]} e^{-\nu_{1} \beta_{n}^{2} t},
\end{aligned}
$$

where $\alpha=\mu_{1} / \mu_{2}, k=\sqrt{\nu_{1} / \nu_{2}}, \sigma=k\left(\mu_{2} / \mu_{1}\right)=k / \alpha$, and $\beta_{n}$ are the positive roots of

$$
\cot \left(\beta_{n} h\right)+\sigma \cot \left(k \beta_{n} a\right)=0
$$

Introducing the following normalized quantities: $\hat{u}=u / U,(\hat{y}, \hat{a})=(y, a) / h, \hat{\beta}_{n}=\beta_{n} h$, and $\hat{t}=t\left(\nu_{1} / h^{2}\right)$, the equations above can be expressed in the following non-dimensional form:

$$
\begin{gathered}
\hat{u}_{1}(\hat{y}, \hat{t})=\frac{\alpha \hat{a}-\hat{y}}{\alpha \hat{a}+1}-2 \sum_{n=1}^{\infty} \frac{\sin ^{2}\left(k \hat{\beta}_{n} \hat{a}\right) \sin \left[\hat{\beta}_{n}(1+\hat{y})\right]}{\hat{\beta}_{n}\left[\sin ^{2}\left(k \hat{\beta}_{n} \hat{a}\right)+\sigma k \hat{a} \sin ^{2}\left(\hat{\beta}_{n}\right)\right]} e^{-\hat{\beta}_{n}^{2} \hat{t}}, \\
\hat{u}_{2}(\hat{y}, \hat{t})=\frac{\alpha(\hat{a}-\hat{y})}{\alpha \hat{a}+1}-2 \sum_{n=1}^{\infty} \frac{\sin \left(\hat{\beta}_{n}\right) \sin \left(k \hat{\beta}_{n} \hat{a}\right) \sin \left[k \hat{\beta}_{n}(\hat{a}-\hat{y})\right]}{\hat{\beta}_{n}\left[\sin ^{2}\left(k \hat{\beta}_{n} \hat{a}\right)+\sigma k \hat{a} \sin ^{2}\left(\hat{\beta}_{n}\right)\right]} e^{-\hat{\beta}_{n}^{2} \hat{t}}, \\
\cot \left(\hat{\beta}_{n}\right)+\sigma \cot \left(k \hat{\beta}_{n} \hat{a}\right)=0 .
\end{gathered}
$$

The rate of flow of the bulk fluid through unit width of the channel is

$$
\hat{q}_{1}(\hat{t})=\int_{-1}^{0} \hat{u}_{1} \mathrm{~d} \hat{y}=\frac{2 \alpha \hat{a}+1}{2(\alpha \hat{a}+1)}-2 \sum_{n=1}^{\infty} \frac{\sin ^{2}\left(k \hat{\beta}_{n} \hat{a}\right)\left[1-\cos \left(\hat{\beta}_{n}\right)\right]}{\hat{\beta}_{n}^{2}\left[\sin ^{2}\left(k \hat{\beta}_{n} \hat{a}\right)+\sigma k \hat{a} \sin ^{2}\left(\hat{\beta}_{n}\right)\right]} e^{-\hat{\beta}_{n}^{2} \hat{t}} .
$$

The depletion layer acts to produce an apparent slip of the bulk fluid at its upper boundary, where the slip is describable by a Navier slip length $\hat{b}(\hat{t})$ given by

$$
\hat{b}(\hat{t})=-\left.\frac{\hat{u}_{1}}{\partial \hat{u}_{1} / \partial \hat{y}}\right|_{\hat{y}=0} .
$$

It is easy to show that, for large times, the limiting slip length is equal to the depletion layer thickness multiplied by the dynamic viscosity ratio:

$$
\hat{b} \rightarrow \hat{b}_{\mathrm{S}}=\alpha \hat{a} \quad \text { as } \hat{t} \rightarrow \infty
$$

which accords with the well-known formula, first reported by Vinogradova [18], for the evaluation of boundary slip due to a low-viscosity depletion layer. As discussed above, $\alpha \gg 1$ 

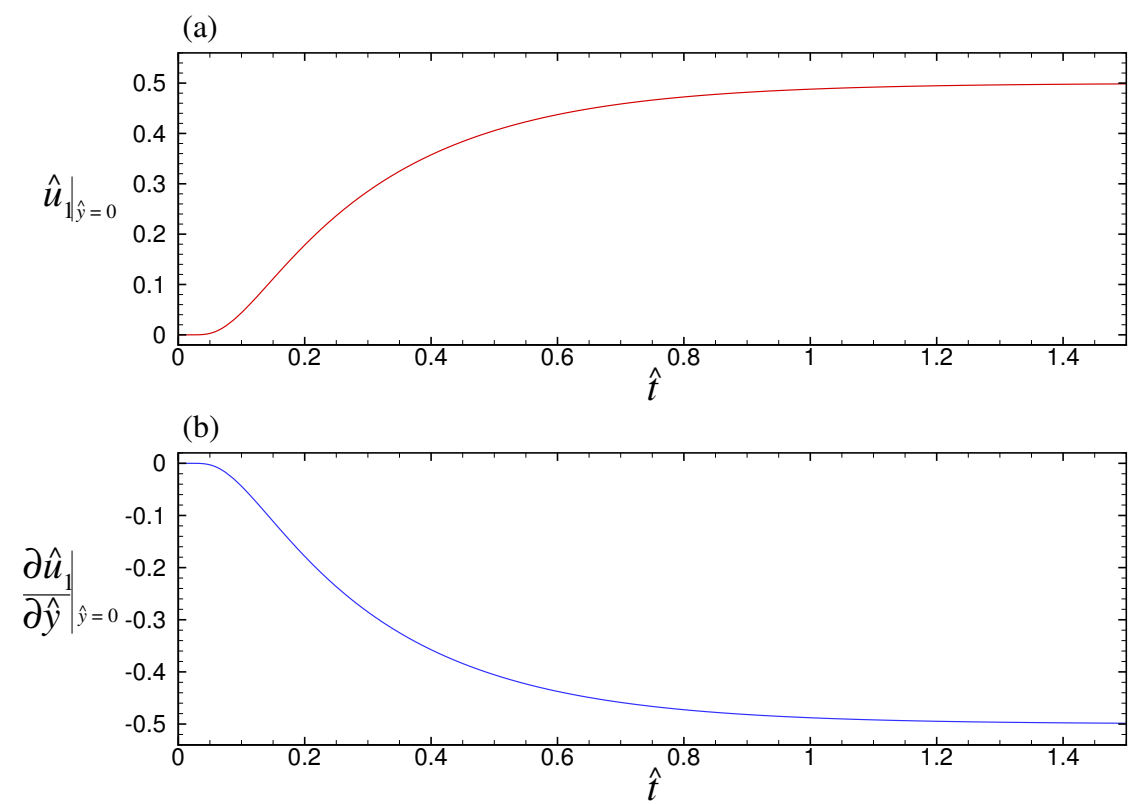

(c)
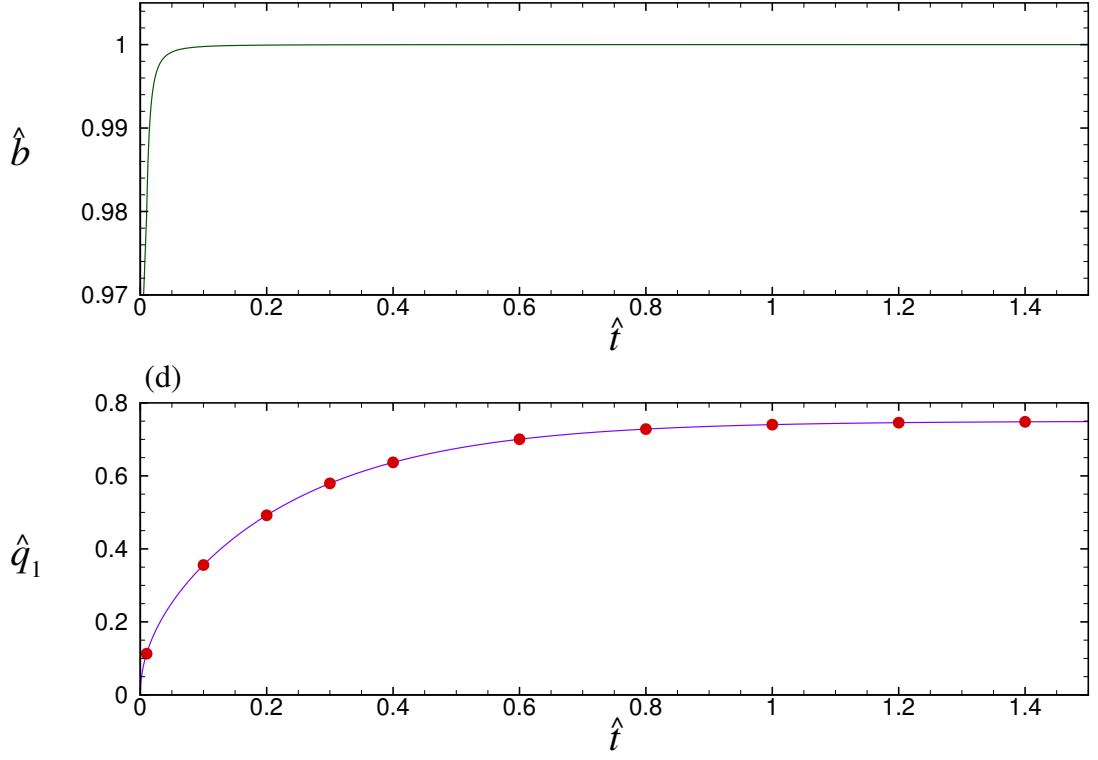

Figure 2: For starting Couette flow with a depletion layer of $k=0.25, \alpha=50$ and $\hat{a}=0.02$ : (a) velocity at the interface $\hat{u}_{1}(\hat{y}=0, \hat{t})$; (b) velocity gradient at the interface $\partial \hat{u}_{1} / \partial \hat{y}(\hat{y}=0, \hat{t})$; (c) slip length $\hat{b}(\hat{t}) ;(\mathrm{d})$ flow rate $\hat{q}_{1}(\hat{t})$. In $(\mathrm{d})$, the symbols denote $\hat{q}$ computed by Eq. (33) for $\hat{b}_{0}=0$ and $\hat{b}_{1}=1$. 
albeit $\hat{a} \ll 1$, and hence $\hat{b}_{\mathrm{S}}$ can be of order unity despite the small thickness of the depletion layer. The question is: how quickly is this steady slip length approached?

Let us for example consider the bulk fluid being an aqueous liquid and the depletion layer being occupied by a gas, for which we may take $k=0.25, \alpha=50$ and $\hat{a}=0.02$. From Eq. (11), we get $\hat{\beta}_{n}=2.0287,4.9131, \cdots$, and from Eq. (14), we get $\hat{b}_{\mathrm{S}}=1$. In Fig. $2(\mathrm{a}, \mathrm{b}, \mathrm{c})$, we show respectively the interfacial velocity $\hat{u}_{1}(\hat{y}=0)$, the velocity gradient at the interface $\partial \hat{u}_{1} / \partial \hat{y}(\hat{y}=0)$, and the slip length $\hat{b}$, as functions of time. Clearly, the startup transient motion will die out in a time period determined by the most slowly decaying exponential, i.e., the exponential containing the leading eigenvalue $\hat{\beta}_{1}$. More precisely, we may estimate that the steady state is practically attained when the time reaches $T_{\mathrm{S}}=5 \hat{\beta}_{1}^{-2}$, as the exponential factor $\exp \left(-\hat{\beta}_{1}^{2} \hat{t}\right)$ becomes smaller than $10^{-2}$ when $\hat{\beta}_{1}^{2} \hat{t}>5$. In this example, $T_{\mathrm{S}}=5 \times 2.0287^{-2}=1.2$, which is indeed the time at which the velocity $\hat{u}_{1}$ and the velocity gradient $\partial \hat{u}_{1} / \partial \hat{y}$ at the interface become very close to their steady-state limits, as shown in Fig. 2(a,b). Nevertheless, the slip length $\hat{b}$, which is equal in magnitude to the ratio of these two quantities, approaches its steady-state limit in a duration much shorter than $T_{\mathrm{S}}$. Figure 2 (c) shows that $\hat{b}$ has already attained $98 \%$ of the limit when $\hat{t}=0.01$. In other words, the slip length will tend to its steady-state limit much faster than the flow itself will do.

On can reason that the quick disappearing of transient for the slip length is due essentially to a much shorter time scale for momentum diffusion across the depletion layer than that across the bulk fluid layer. This ratio, given by $\left(a^{2} / \nu_{2}\right) /\left(h^{2} / \nu_{1}\right)=(k \hat{a})^{2}$, is much less than unity in this example.

On ignoring the short-lived transient for the boundary slip, we may simplify the problem by introducing a Navier slip condition to substitute for the action of the depletion layer:

$$
\hat{u}_{1}=-\hat{b}_{1} \frac{\partial \hat{u}_{1}}{\partial \hat{y}} \quad \text { at } \hat{y}=0
$$

where the slip length $\hat{b}_{1}$ is a constant. Such a problem with constant slip lengths is described in Section 3. As shown in Fig. 2(d), the flow rate $\hat{q}_{1}(\hat{t})$ computed from Eq. (12) is virtually the same as the flow rate $\hat{q}(\hat{t})$ computed from Eq. (33) for $\hat{b}_{0}=0$ and $\hat{b}_{1}=1$.

In the following problems, we shall use the Navier slip condition with a time-invariant slip length to model the boundary slip. 


\section{Plane Couette flow between two plates}

We now consider starting Couette flow between two parallel flat plates, at a distance $h$ apart, with constant but disparate slip lengths $b_{0}$ and $b_{1}$ on the lower and upper boundaries; see Fig. 3. The lower plate is suddenly set into motion at a constant velocity $U$ in its own plane. The problem is as follows:

$$
\begin{gathered}
\frac{\partial u}{\partial t}=\nu \frac{\partial^{2} u}{\partial y^{2}} \quad \text { in } 0 \leq y \leq h, \\
u=\left\{\begin{array}{ll}
U+b_{0} \frac{\partial u}{\partial y}, & t>0 \\
0, & t<0
\end{array} \text { at } y=0,\right. \\
u=-b_{1} \frac{\partial u}{\partial y} \quad \text { at } y=h .
\end{gathered}
$$

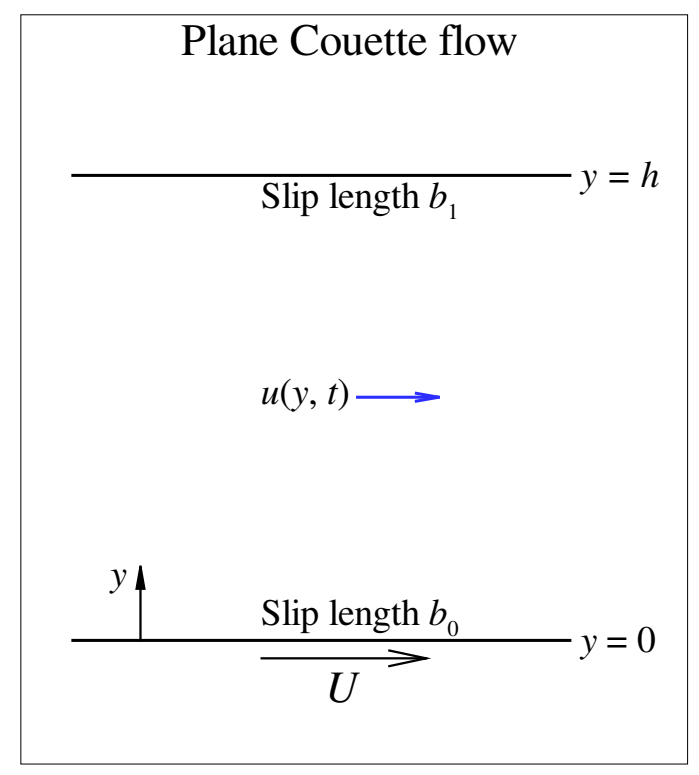

Figure 3: Starting Couette flow between two parallel flat plates.

We first decompose the velocity into steady and transient parts:

$$
u(y, t)=u_{\mathrm{S}}(y)-u_{\mathrm{T}}(y, t),
$$

where the steady-state velocity is

$$
u_{\mathrm{S}}(y)=\frac{U h}{h+b_{0}+b_{1}}\left(1+\frac{b_{1}}{h}-\frac{y}{h}\right),
$$


and the transient velocity is governed by

$$
\begin{gathered}
\frac{\partial u_{\mathrm{T}}}{\partial t}=\nu \frac{\partial^{2} u_{\mathrm{T}}}{\partial y^{2}} \quad \text { in } 0 \leq y \leq h, \\
u_{\mathrm{T}}=b_{0} \frac{\partial u_{\mathrm{T}}}{\partial y} \quad \text { at } y=0, \\
u_{\mathrm{T}}=-b_{1} \frac{\partial u_{\mathrm{T}}}{\partial y} \quad \text { at } y=h, \\
u_{\mathrm{T}} \rightarrow 0 \quad \text { as } t \rightarrow \infty \\
u_{\mathrm{T}}=u_{\mathrm{S}} \quad \text { at } t=0 .
\end{gathered}
$$

The solution to Eqs. (21)-(24) can be obtained by eigenfunction expansions:

$$
u_{\mathrm{T}}(y, t)=\sum_{n=1}^{\infty} A_{n}\left[\sin \left(\beta_{n} y\right)+\beta_{n} b_{0} \cos \left(\beta_{n} y\right)\right] e^{-\nu \beta_{n}^{2} t},
$$

where $\beta_{n}$ are eigenvalues given by the positive roots of the characteristic equation

$$
\left(1-\beta_{n}^{2} b_{0} b_{1}\right) \sin \left(\beta_{n} h\right)+\beta_{n}\left(b_{0}+b_{1}\right) \cos \left(\beta_{n} h\right)=0 .
$$

The coefficients $A_{n}$ are determined from the initial condition, Eq. (25),

$$
\sum_{n=1}^{\infty} A_{n}\left[\sin \left(\beta_{n} y\right)+\beta_{n} b_{0} \cos \left(\beta_{n} y\right)\right]=\frac{U h}{h+b_{0}+b_{1}}\left(1+\frac{b_{1}}{h}-\frac{y}{h}\right)
$$

Using orthogonality of the eigenfunctions, we get

$$
A_{n}=\frac{4 U}{\left(1+\beta_{n}^{2} b_{0}^{2}\right) 2 \beta_{n} h-\left(1-\beta_{n}^{2} b_{0}^{2}\right) \sin \left(2 \beta_{n} h\right)+4 \beta_{n} b_{0} \sin ^{2}\left(\beta_{n} h\right)} .
$$

In terms of normalized quantities: $\left(\hat{u}, \hat{A}_{n}\right)=\left(u, A_{n}\right) / U,(\hat{y}, \hat{b})=(y, b) / h, \hat{\beta}_{n}=\beta_{n} h$, and $\hat{t}=t\left(\nu / h^{2}\right)$, the velocity for the present problem in dimensionless form is

$$
\hat{u}(\hat{y}, \hat{t})=\frac{1+\hat{b}_{1}-\hat{y}}{1+\hat{b}_{0}+\hat{b}_{1}}-\sum_{n=1}^{\infty} \hat{A}_{n}\left[\sin \left(\hat{\beta}_{n} \hat{y}\right)+\hat{\beta}_{n} \hat{b}_{0} \cos \left(\hat{\beta}_{n} \hat{y}\right)\right] e^{-\hat{\beta}_{n}^{2} \hat{t}}
$$

where

$$
\hat{A}_{n}=\frac{4}{\left(1+\hat{\beta}_{n}^{2} \hat{b}_{0}^{2}\right) 2 \hat{\beta}_{n}-\left(1-\hat{\beta}_{n}^{2} \hat{b}_{0}^{2}\right) \sin \left(2 \hat{\beta}_{n}\right)+4 \hat{\beta}_{n} \hat{b}_{0} \sin ^{2}\left(\hat{\beta}_{n}\right)},
$$

and $\hat{\beta}_{n}$ are the positive roots of

$$
\left(1-\hat{\beta}_{n}^{2} \hat{b}_{0} \hat{b}_{1}\right) \sin \left(\hat{\beta}_{n}\right)+\hat{\beta}_{n}\left(\hat{b}_{0}+\hat{b}_{1}\right) \cos \left(\hat{\beta}_{n}\right)=0
$$


The rate of flow through unit width of the channel is

$$
\hat{q}(\hat{t})=\int_{0}^{1} \hat{u} \mathrm{~d} \hat{y}=\frac{2 \hat{b}_{1}+1}{2\left(1+\hat{b}_{0}+\hat{b}_{1}\right)}-\sum_{n=1}^{\infty} \frac{\hat{A}_{n}}{\hat{\beta}_{n}}\left[1-\cos \left(\hat{\beta}_{n}\right)+\hat{\beta}_{n} \hat{b}_{0} \sin \left(\hat{\beta}_{n}\right)\right] e^{-\hat{\beta}_{n}^{2} \hat{t}} .
$$

When the two slip lengths are the same, $\hat{b}_{0}=\hat{b}_{1}=\hat{b}$, Eqs. (30), (32) and (33) simplify to

$$
\begin{gathered}
\hat{u}(\hat{y}, \hat{t})=\frac{1+\hat{b}-\hat{y}}{1+2 \hat{b}}-2 \sum_{n=1}^{\infty} \frac{\sin \left(\hat{\beta}_{n} \hat{y}\right)+\hat{\beta}_{n} \hat{b} \cos \left(\hat{\beta}_{n} \hat{y}\right)}{\hat{\beta}_{n}\left(1+2 \hat{b}+\hat{\beta}_{n}^{2} \hat{b}^{2}\right)} e^{-\hat{\beta}_{n}^{2} \hat{t}}, \\
\left(1-\hat{\beta}_{n}^{2} \hat{b}^{2}\right) \sin \left(\hat{\beta}_{n}\right)+2 \hat{\beta}_{n} \hat{b} \cos \left(\hat{\beta}_{n}\right)=0,
\end{gathered}
$$

and

$$
\hat{q}(\hat{t})=\frac{1}{2}-2 \sum_{n=1}^{\infty} \frac{1-\cos \left(\hat{\beta}_{n}\right)+\hat{\beta}_{n} \hat{b} \sin \left(\hat{\beta}_{n}\right)}{\hat{\beta}_{n}^{2}\left(1+2 \hat{b}+\hat{\beta}_{n}^{2} \hat{b}^{2}\right)} e^{-\hat{\beta}_{n}^{2} \hat{t}} .
$$

Equations (34) and (35) can be checked to agree with those deduced by Kaoullas and Georgiou [14], upon omitting the dynamic slip and rectifying some typographical errors in their equations.

For no-slip walls, $\hat{b}=0$, the eigenvalues are $\hat{\beta}_{n}=n \pi$, by which Eqs. (34) and (36) further simplify to

$$
\hat{u}_{\text {no-slip }}=1-\hat{y}-2 \sum_{n=1}^{\infty} \frac{\sin (n \pi \hat{y})}{n \pi} e^{-n^{2} \pi^{2} \hat{t}}
$$

and

$$
\hat{q}_{\text {no-slip }}=\frac{1}{2}-2 \sum_{n=1}^{\infty} \frac{\left[1+(-1)^{n+1}\right]}{n^{2} \pi^{2}} e^{-n^{2} \pi^{2} \hat{t}} .
$$

Equation (37) is well known in the literature; see White [19] and Leal [20].

We have solved Eq. (32), and other characteristic equations to be introduced in the following sections, with the aid of Mathematica. For future reference, we provide in Table A1 the first five eigenvalues $\hat{\beta}_{n}$ and Fourier coefficients $\hat{A}_{n}$ for some values of $\hat{b}_{0}$ and $\hat{b}_{1}$. For fixed $\hat{b}_{1}$, the coefficients $\hat{A}_{n}$ drop to zero faster with increasing $n$ under a non-zero value of $\hat{b}_{0}$, suggesting that the series solution may converge with fewer terms in the presence of slip on the bottom wall. The presence of slip on the top wall, however, has no similar effect. Despite this effect of slip on the Fourier coefficients, we have computed the series solutions using at least 20 terms to ensure sufficient accuracy of the solutions, especially for small times.

In Fig. 4 , we show the leading eigenvalue $\hat{\beta}_{1}$ as a function of $\hat{b}_{1}$ for $\hat{b}_{0}=0,1$. As discussed above, the time scale for the slowest decaying transient of the starting flow is determined 


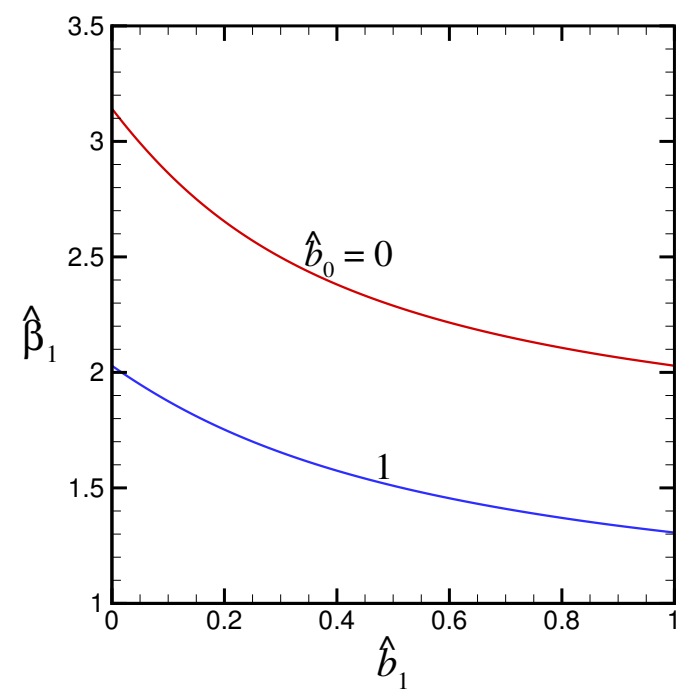

Figure 4: For starting plane Couette flow, the leading eigenvalue $\hat{\beta}_{1}$ as a function of the slip lengths $\hat{b}_{0}$ and $\hat{b}_{1}$.

by $\hat{\beta}_{1}^{-2}$. Hence, the smaller $\hat{\beta}_{1}$, the longer the transient period. The figure clearly shows that the presence of boundary slip will in general lengthen the transient period. When both boundaries are non-slipping $\hat{b}_{0}=\hat{b}_{1}=0, \hat{\beta}_{1}=\pi$. When both boundaries are slipping with $\hat{b}_{0}=\hat{b}_{1}=1$, the leading eigenvalue is $\hat{\beta}_{1}=1.3065$, which implies that the transient period will be approximately $(\pi / 1.3065)^{2}=5.8$ times longer than that when both boundaries are non-slipping.

The effect of the boundary slip on the starting flow is further illustrated in Fig. 5, where we show the velocity profile as a function of time for four sets of values of the slip lengths. Without any boundary slip $\left(\hat{b}_{0}=\hat{b}_{1}=0\right)$, the steady state is quickly attained. With slip, it takes some time for the velocity at the slipping wall to reach its steady-state value. Therefore, the transient motion may persist for a longer time when both boundaries are slipping. This prolonged transient period owes its origin to the fact that boundary slip is to retard the rate of momentum transfer by diffusion across the boundary where the slip occurs. 

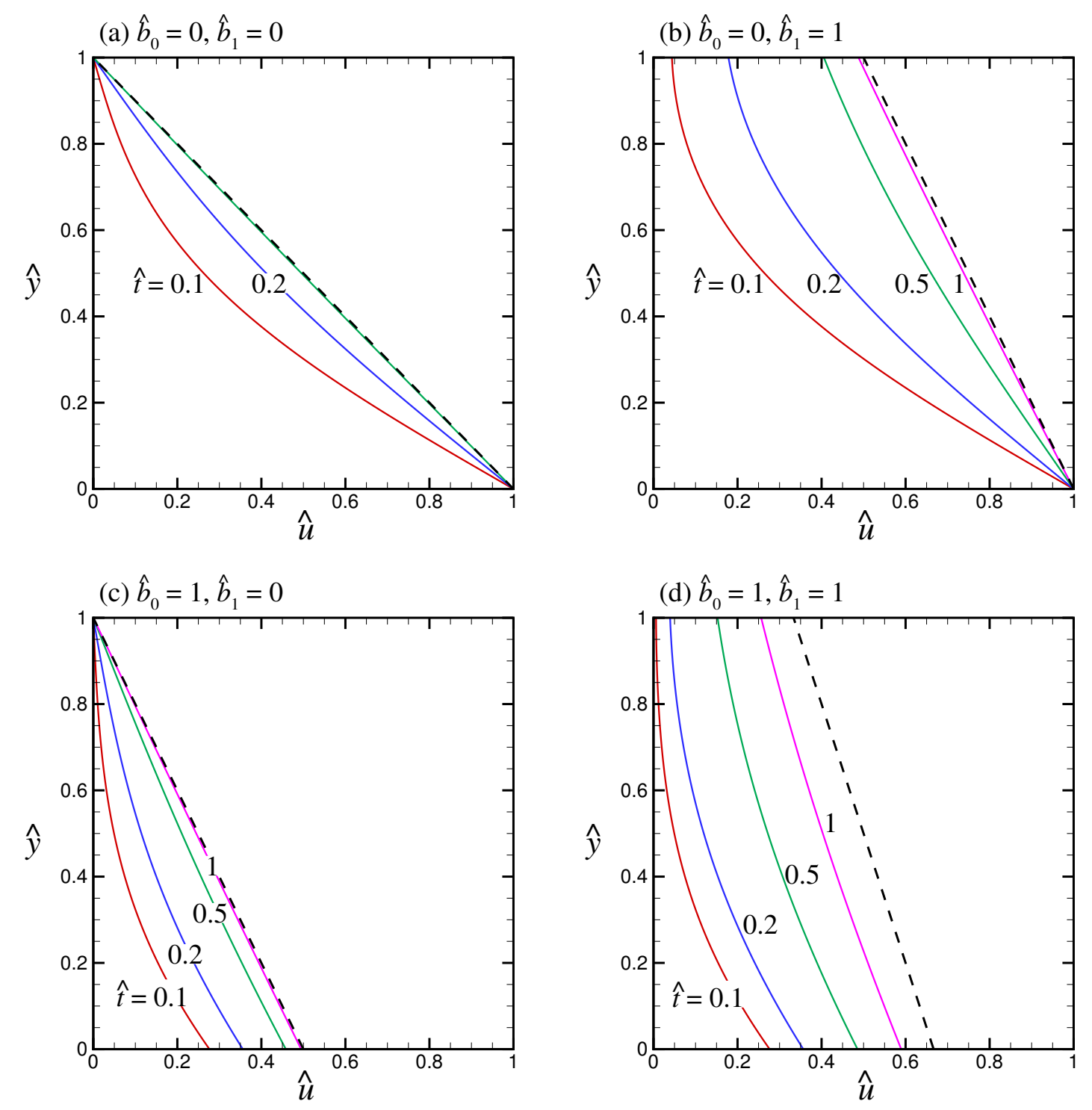

Figure 5: For starting plane Couette flow, the velocity profile $\hat{u}(\hat{y})$ as a function of $\hat{t}$, for (a) $\hat{b}_{0}=0, \hat{b}_{1}=0 ;(\mathrm{b}) \hat{b}_{0}=0, \hat{b}_{1}=1 ;$ (c) $\hat{b}_{0}=1, \hat{b}_{1}=0 ;$ (d) $\hat{b}_{0}=1, \hat{b}_{1}=1$. The dashed lines denote the steady-state velocity profiles. 


\section{Rotary Couette flow between two cylinders}

We next consider starting Couette flow between two rotating coaxial cylinders, where the inner and outer cylinders have radii $R_{0}$ and $R_{1}$, and slip lengths $b_{0}$ and $b_{1}$ on their surfaces, respectively, see Fig. 6. The problem is as follows:

$$
\frac{\partial u}{\partial t}=\nu \frac{\partial}{\partial r}\left[\frac{1}{r} \frac{\partial}{\partial r}(r u)\right],
$$

where $u(r, t)$ is the azimuthal velocity, subject to the following boundary conditions:

$$
\begin{aligned}
& u=\left\{\begin{array}{ll}
U_{0}+b_{0}\left(\frac{\partial u}{\partial r}-\frac{u}{R_{0}}\right), & t>0 \\
0, & t<0
\end{array} \quad \text { at } r=R_{0},\right. \\
& u=\left\{\begin{array}{ll}
U_{1}-b_{1}\left(\frac{\partial u}{\partial r}-\frac{u}{R_{1}}\right), & t>0 \\
0, & t<0
\end{array} \quad \text { at } r=R_{1} .\right.
\end{aligned}
$$

Here, for generality, we allow impulsively started azimuthal flow to be caused by sudden

rotation of either cylinder or both cylinders. When started, $U_{0}$ and $U_{1}$ are the azimuthal velocities of the inner and outer boundaries of the annulus. In the slip conditions above, use has been made of the following relationship for shear stress tangential to a cylindrical surface

$$
\tau_{r \theta}=\mu r \frac{\partial}{\partial r}\left(\frac{u}{r}\right)=\mu\left(\frac{\partial u}{\partial r}-\frac{u}{r}\right) .
$$

We first decompose the velocity into steady and transient components

$$
u(r, t)=u_{\mathrm{S}}(r)-u_{\mathrm{T}}(r, t)
$$

where $u_{\mathrm{T}}(t=0)=u_{\mathrm{S}}$ and $u_{\mathrm{T}}(t \rightarrow \infty) \rightarrow 0$. The steady-state velocity satisfying the slip boundary conditions is readily found to be

$$
u_{\mathrm{S}}(r)=c_{1} r+\frac{c_{2}}{r},
$$

where

$$
\begin{gathered}
c_{1}=\frac{R_{0}^{2}\left(R_{1}-2 b_{1}\right) U_{0}-R_{1}^{2}\left(R_{0}+2 b_{0}\right) U_{1}}{R_{0}^{3}\left(R_{1}-2 b_{1}\right)-R_{1}^{3}\left(R_{0}+2 b_{0}\right)}, \\
c_{2}=\frac{R_{0}^{2} R_{1}^{2}\left(-R_{1} U_{0}+R_{0} U_{1}\right)}{R_{0}^{3}\left(R_{1}-2 b_{1}\right)-R_{1}^{3}\left(R_{0}+2 b_{0}\right)} .
\end{gathered}
$$




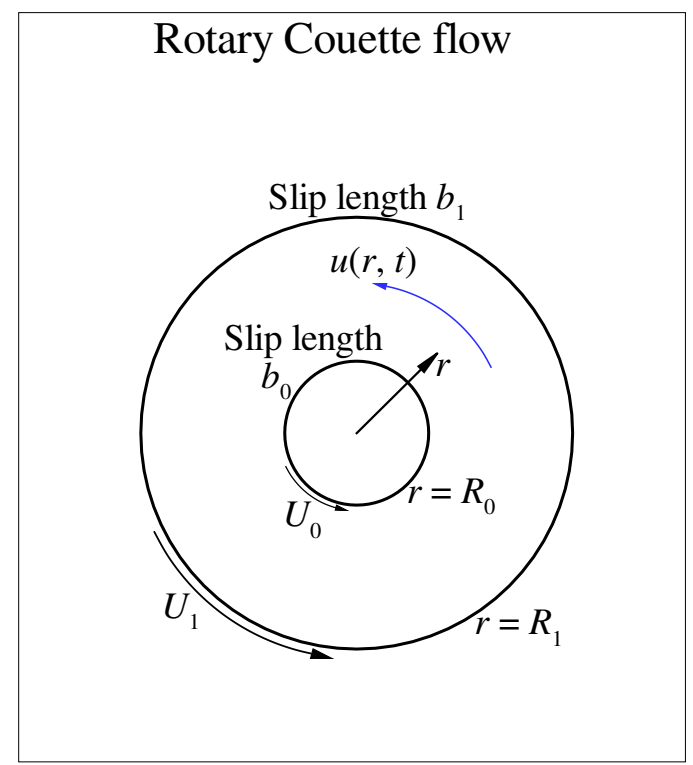

Figure 6: Starting Couette flow between two rotating coaxial cylinders.

Applying the method of eigenfunction expansions, the transient velocity that satisfies the momentum equation and the boundary conditions without the forcing can be found as follows:

$$
u_{\mathrm{T}}(r, t)=\sum_{n=1}^{\infty} A_{n} Z_{1}\left(\beta_{n} r\right) e^{-\nu \beta_{n}^{2} t}
$$

where

$$
Z_{k}\left(\beta_{n} r\right) \equiv J_{k}\left(\beta_{n} r\right)+\lambda_{n} Y_{k}\left(\beta_{n} r\right)
$$

in which $J_{k}$ and $Y_{k}$ are respectively the Bessel functions of the first and the second kinds of order $k$, and

$$
\lambda_{n}=-\frac{\left(1+2 b_{0} / R_{0}\right) J_{1}\left(\beta_{n} R_{0}\right)-\beta_{n} b_{0} J_{0}\left(\beta_{n} R_{0}\right)}{\left(1+2 b_{0} / R_{0}\right) Y_{1}\left(\beta_{n} R_{0}\right)-\beta_{n} b_{0} Y_{0}\left(\beta_{n} R_{0}\right)} .
$$

The eigenvalues $\beta_{n}$ are determined by the following characteristic equation

$$
\begin{aligned}
& {\left[\left(1-2 b_{1} / R_{1}\right) J_{1}\left(\beta_{n} R_{1}\right)+\beta_{n} b_{1} J_{0}\left(\beta_{n} R_{1}\right)\right]\left[\left(1+2 b_{0} / R_{0}\right) Y_{1}\left(\beta_{n} R_{0}\right)-\beta_{n} b_{0} Y_{0}\left(\beta_{n} R_{0}\right)\right]} \\
& \quad-\left[\left(1-2 b_{1} / R_{1}\right) Y_{1}\left(\beta_{n} R_{1}\right)+\beta_{n} b_{1} Y_{0}\left(\beta_{n} R_{1}\right)\right]\left[\left(1+2 b_{0} / R_{0}\right) J_{1}\left(\beta_{n} R_{0}\right)-\beta_{n} b_{0} J_{0}\left(\beta_{n} R_{0}\right)\right] \\
& \quad=0 .
\end{aligned}
$$

The coefficients $A_{n}$ are found from the initial condition $u_{\mathrm{T}}(t=0)=u_{\mathrm{S}}$. Using orthogonality of the eigenfunctions (with $r$ being the weighting function), we may obtain after some 
algebra:

$$
A_{n}=\frac{D_{n}}{E_{n}}
$$

where

$$
\begin{aligned}
& D_{n}= \frac{R_{0}\left[J_{0}\left(\beta_{n} R_{0}\right) Y_{1}\left(\beta_{n} R_{0}\right)-J_{1}\left(\beta_{n} R_{0}\right) Y_{0}\left(\beta_{n} R_{0}\right)\right] U_{0}}{\beta_{n}\left[\left(1+2 b_{0} / R_{0}\right) Y_{1}\left(\beta_{n} R_{0}\right)-\beta_{n} b_{0} Y_{0}\left(\beta_{n} R_{0}\right)\right]} \\
&+\frac{R_{1}\left[J_{1}\left(\beta_{n} R_{1}\right) Y_{0}\left(\beta_{n} R_{1}\right)-J_{0}\left(\beta_{n} R_{1}\right) Y_{1}\left(\beta_{n} R_{1}\right)\right] U_{1}}{\beta_{n}\left[\left(1-2 b_{1} / R_{1}\right) Y_{1}\left(\beta_{n} R_{1}\right)+\beta_{n} b_{1} Y_{0}\left(\beta_{n} R_{1}\right)\right]}, \\
& E_{n}=\left[\frac{r^{2}}{2}\left[Z_{0}^{2}\left(\beta_{n} r\right)+Z_{1}^{2}\left(\beta_{n} r\right)\right]-\frac{r}{\beta_{n}} Z_{0}\left(\beta_{n} r\right) Z_{1}\left(\beta_{n} r\right)\right]_{R_{0}}^{R_{1}} .
\end{aligned}
$$

If $U_{0} \neq 0$, we may introduce the following normalization: $\left(\hat{u}, \hat{U}_{1}, \hat{A}_{n}\right)=\left(u, U_{1}, A_{n}\right) / U_{0}$, $\left(\hat{r}, \hat{b}, \hat{R}_{0}\right)=\left(r, b, R_{0}\right) / R_{1}, \hat{\beta}_{n}=\beta_{n} R_{1}$, and $\hat{t}=t\left(\nu / R_{1}^{2}\right)$. The non-dimensional velocity for the present problem then reads as

$$
\hat{u}(\hat{r}, \hat{t})=\hat{c}_{1} \hat{r}+\frac{\hat{c}_{2}}{\hat{r}}-\sum_{n=1}^{\infty} \hat{A}_{n} Z_{1}\left(\hat{\beta}_{n} \hat{r}\right) e^{-\hat{\beta}_{n}^{2} \hat{t}}
$$

where

$$
\begin{gathered}
\hat{c}_{1}=\frac{\hat{R}_{0}^{2}\left(1-2 \hat{b}_{1}\right)-\left(\hat{R}_{0}+2 \hat{b}_{0}\right) \hat{U}_{1}}{\hat{R}_{0}^{3}\left(1-2 \hat{b}_{1}\right)-\left(\hat{R}_{0}+2 \hat{b}_{0}\right)}, \\
\hat{c}_{2}=\frac{\hat{R}_{0}^{2}\left(-1+\hat{R}_{0} \hat{U}_{1}\right)}{\hat{R}_{0}^{3}\left(1-2 \hat{b}_{1}\right)-\left(\hat{R}_{0}+2 \hat{b}_{0}\right)},
\end{gathered}
$$

and $\hat{\beta}_{n}$ are the positive roots of

$$
\begin{aligned}
& {\left[\left(1-2 \hat{b}_{1}\right) J_{1}\left(\hat{\beta}_{n}\right)+\hat{\beta}_{n} \hat{b}_{1} J_{0}\left(\hat{\beta}_{n}\right)\right]\left[\left(1+2 \hat{b}_{0} / \hat{R}_{0}\right) Y_{1}\left(\hat{\beta}_{n} \hat{R}_{0}\right)-\hat{\beta}_{n} \hat{b}_{0} Y_{0}\left(\hat{\beta}_{n} \hat{R}_{0}\right)\right]} \\
& \quad-\left[\left(1-2 \hat{b}_{1}\right) Y_{1}\left(\hat{\beta}_{n}\right)+\hat{\beta}_{n} \hat{b}_{1} Y_{0}\left(\hat{\beta}_{n}\right)\right]\left[\left(1+2 \hat{b}_{0} / \hat{R}_{0}\right) J_{1}\left(\hat{\beta}_{n} \hat{R}_{0}\right)-\hat{\beta}_{n} \hat{b}_{0} J_{0}\left(\hat{\beta}_{n} \hat{R}_{0}\right)\right] \\
& \quad=0 .
\end{aligned}
$$

The non-dimensional coefficients $\hat{A}_{n}$ may be expressed likewise. For future reference, we provide in Table A2 the first five eigenvalues $\hat{\beta}_{n}$ for $\hat{R}_{0}=0.1,0.5$, and several values of $\hat{b}_{0}$ and $\hat{b}_{1}$.

When the outer cylinder is stationary $\hat{U}_{1}=0$ and the two slip lengths are the same $\hat{b}_{0}=\hat{b}_{1}$, our problem becomes the one studied by Kaoullas and Georgiou [14] without the dynamic slip. Figure 7 shows the agreement of velocity profiles computed by their and our solutions, thereby validating our equations deduced above. 

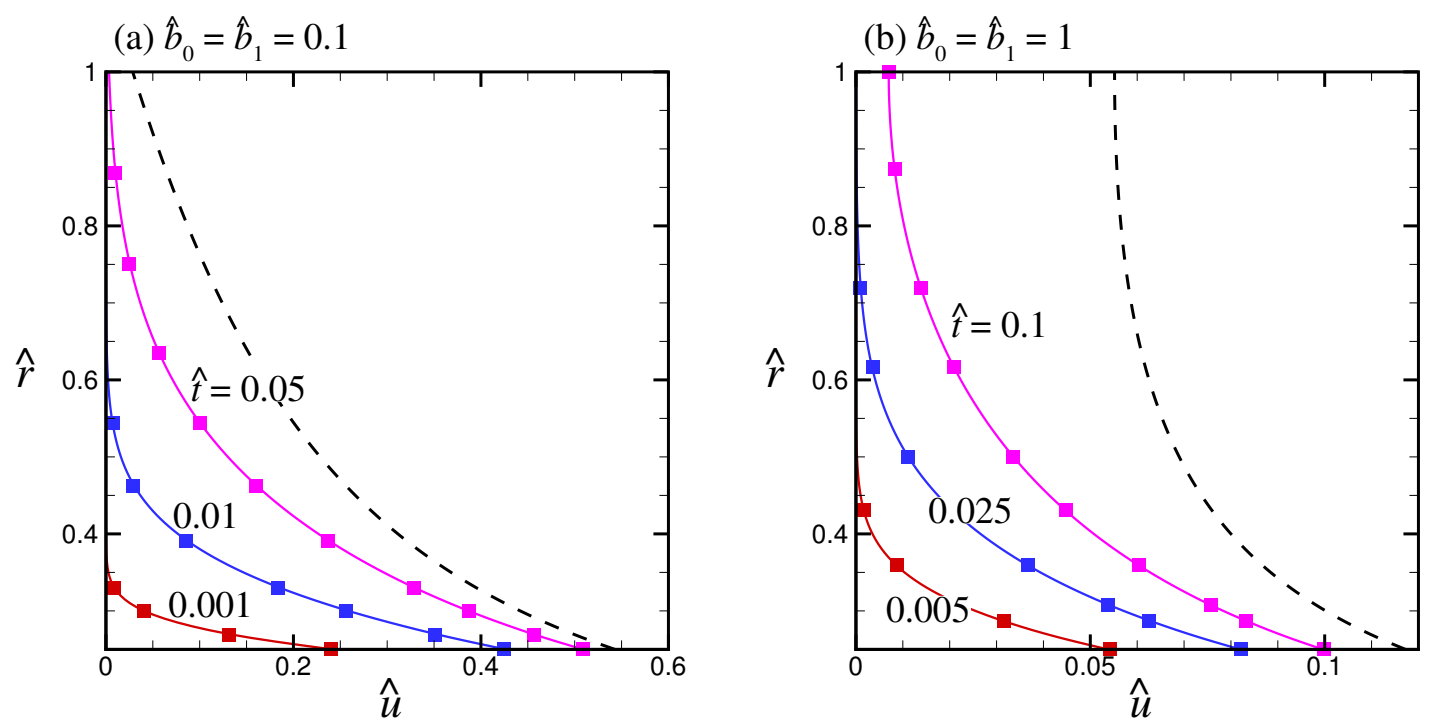

Figure 7: For starting Couette flow between two coaxial cylinders, the azimuthal velocity profile $\hat{u}(\hat{r})$ at various times, for $\hat{U}_{1}=0, \hat{R}_{0}=0.25$, and (a) $\hat{b}_{0}=\hat{b}_{1}=0.1$; (b) $\hat{b}_{0}=\hat{b}_{1}=1$. The dashed lines denote the steady-state velocity profiles. The square symbols are results computed by Kaoullas and Georgiou [14].

\section{Poiseuille flow through a parallel-plate channel}

We next consider starting pressure-driven flow through a slit channel of height $h$, where the slip lengths on the lower and upper walls are $b_{0}$ and $b_{1}$, respectively; see Fig. 8 . The problem is as follows:

$$
\frac{\partial u}{\partial t}=-\frac{1}{\rho} \frac{\partial p}{\partial x}+\nu \frac{\partial^{2} u}{\partial y^{2}} \quad \text { in } 0 \leq y \leq h,
$$

where $u(y, t)$ is the axial velocity, and the axial pressure gradient is

$$
-\frac{\partial p}{\partial x}=\left\{\begin{array}{cc}
K, & t>0 \\
0, & t<0
\end{array}\right.
$$

in which $K$ is the pressure gradient suddenly applied to the fluid. The boundary conditions are

$$
\begin{array}{cl}
u=b_{0} \frac{\partial u}{\partial y} & \text { at } y=0, \\
u=-b_{1} \frac{\partial u}{\partial y} & \text { at } y=h
\end{array}
$$


The problem formulated above and the solution deduced below are essentially the same as those given by Matthews and Hastie [13], but are presented here in terms of the present notation for completeness of this paper. The same problem but under the condition of equal slip lengths on the two walls has been solved by Avramenko et al. [11] using Laplace transform. We shall express the solution similar to the one by Kaoullas and Georgiou [14], which is more compact in form than the solution given by Avramenko et al. [11].

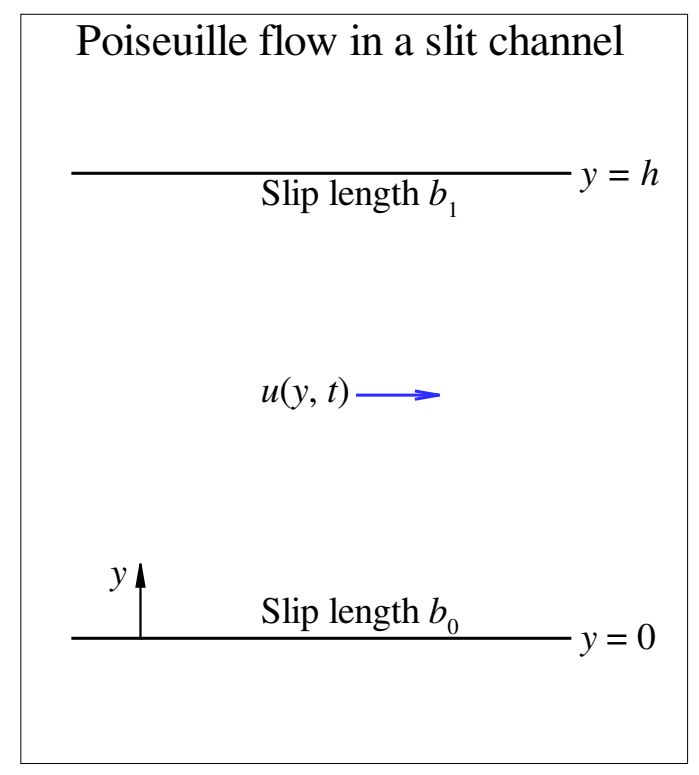

Figure 8: Starting Poiseuille flow through a slit channel bounded by two parallel flat plates.

We first decompose the velocity into steady and transient components

$$
u(y, t)=u_{\mathrm{S}}(y)-u_{\mathrm{T}}(y, t),
$$

where $u_{\mathrm{T}}$ is initially equal to $u_{\mathrm{S}}$ and decays to zero for large times. The steady-state velocity is readily found to be

$$
u_{\mathrm{S}}(y)=\frac{K h^{2}}{2 \mu}\left[-\frac{y^{2}}{h^{2}}+\frac{\left(h+2 b_{1}\right)\left(y+b_{0}\right)}{\left(h+b_{0}+b_{1}\right) h}\right],
$$

and the transient velocity is expressible by the same eigenfunctions as in the plane Couette flow problem:

$$
u_{\mathrm{T}}(y, t)=\sum_{n=1}^{\infty} A_{n}\left[\sin \left(\beta_{n} y\right)+\beta_{n} b_{0} \cos \left(\beta_{n} y\right)\right] e^{-\nu \beta_{n}^{2} t}
$$


where $\beta_{n}$ are the positive roots of

$$
\left(1-\beta_{n}^{2} b_{0} b_{1}\right) \sin \left(\beta_{n} h\right)+\beta_{n}\left(b_{0}+b_{1}\right) \cos \left(\beta_{n} h\right)=0
$$

which is identical to Eq. (27), the characteristic equation for Couette flow between two plates. The coefficients $A_{n}$ are determined from the initial condition $u_{\mathrm{T}}(t=0)=u_{\mathrm{S}}$. Using orthogonality of the eigenfunctions, we can get

$$
A_{n}=\frac{4 K\left[\beta_{n} b_{0} \sin \left(\beta_{n} h\right)+1-\cos \left(\beta_{n} h\right)\right]}{\mu \beta_{n}^{2}\left[\left(1+\beta_{n}^{2} b_{0}^{2}\right) 2 \beta_{n} h-\left(1-\beta_{n}^{2} b_{0}^{2}\right) \sin \left(2 \beta_{n} h\right)+4 \beta_{n} b_{0} \sin ^{2}\left(\beta_{n} h\right)\right]} .
$$

In terms of normalized quantities: $\left(\hat{u}, \hat{A}_{n}\right)=\left(u, A_{n}\right) /\left(K h^{2} / \mu\right),(\hat{y}, \hat{b})=(y, b) / h, \hat{\beta}_{n}=\beta_{n} h$, and $\hat{t}=t\left(\nu / h^{2}\right)$, the velocity for the present problem in dimensionless form is

$$
\hat{u}(\hat{y}, \hat{t})=\frac{1}{2}\left[-\hat{y}^{2}+\frac{\left(1+2 \hat{b}_{1}\right)\left(\hat{y}+\hat{b}_{0}\right)}{\left(1+\hat{b}_{0}+\hat{b}_{1}\right)}\right]-\sum_{n=1}^{\infty} \hat{A}_{n}\left[\sin \left(\hat{\beta}_{n} \hat{y}\right)+\hat{\beta}_{n} \hat{b}_{0} \cos \left(\hat{\beta}_{n} \hat{y}\right)\right] e^{-\hat{\beta}_{n}^{2} \hat{t}},
$$

where

$$
\hat{A}_{n}=\frac{4\left[\hat{\beta}_{n} \hat{b}_{0} \sin \left(\hat{\beta}_{n}\right)+1-\cos \left(\hat{\beta}_{n}\right)\right]}{\hat{\beta}_{n}^{2}\left[\left(1+\hat{\beta}_{n}^{2} \hat{b}_{0}^{2}\right) 2 \hat{\beta}_{n}-\left(1-\hat{\beta}_{n}^{2} \hat{b}_{0}^{2}\right) \sin \left(2 \hat{\beta}_{n}\right)+4 \hat{\beta}_{n} \hat{b}_{0} \sin ^{2}\left(\hat{\beta}_{n}\right)\right]},
$$

and $\hat{\beta}_{n}$ are the positive roots of

$$
\left(1-\hat{\beta}_{n}^{2} \hat{b}_{0} \hat{b}_{1}\right) \sin \left(\hat{\beta}_{n}\right)+\hat{\beta}_{n}\left(\hat{b}_{0}+\hat{b}_{1}\right) \cos \left(\hat{\beta}_{n}\right)=0
$$

The rate of flow through unit width of the channel is

$$
\hat{q}(\hat{t})=\int_{0}^{1} \hat{u} \mathrm{~d} \hat{y}=\frac{\left(1+2 \hat{b}_{0}\right)\left(1+2 \hat{b}_{1}\right)}{4\left(1+\hat{b}_{0}+\hat{b}_{1}\right)}-\frac{1}{6}-\sum_{n=1}^{\infty} \frac{\hat{A}_{n}}{\hat{\beta}_{n}}\left[1-\cos \left(\hat{\beta}_{n}\right)+\hat{\beta}_{n} \hat{b}_{0} \sin \left(\hat{\beta}_{n}\right)\right] e^{-\hat{\beta}_{n}^{2} \hat{t}}
$$

For no-slip walls, $\hat{b}_{0}=\hat{b}_{1}=0$, we get $\hat{\beta}_{n}=n \pi$, and $\hat{A}_{n}=2\left[1+(-1)^{n+1}\right] /(n \pi)^{3}$. Equations (67) and (70) then simplify to

$$
\begin{gathered}
\hat{u}_{\text {no-slip }}=\frac{1}{2}\left(\hat{y}-\hat{y}^{2}\right)-2 \sum_{n=1}^{\infty} \frac{\left[1+(-1)^{n+1}\right]}{n^{3} \pi^{3}} \sin (n \pi \hat{y}) e^{-n^{2} \pi^{2} \hat{t}}, \\
\hat{q}_{\text {no-slip }}=\frac{1}{12}-2 \sum_{n=1}^{\infty} \frac{\left[1+(-1)^{n+1}\right]^{2}}{n^{4} \pi^{4}} e^{-n^{2} \pi^{2} \hat{t}} .
\end{gathered}
$$

After reconciling the normalization scales, the expressions given above can be checked to agree with those presented by Matthews and Hastie [13]. Our expression above for the Fourier coefficients $\hat{A}_{n}$ is slightly simpler in form than theirs. 
One may note that the terms in the series above for the no-slip case vanish for even values of $n$. The particular case when the two slip lengths are the same, $\hat{b}_{0}=\hat{b}_{1}=\hat{b}$, warrants some further discussion. Under this special condition, the characteristic equation, Eq. (69), can be factorized into

$$
\left[\cos \left(\hat{\beta}_{n} / 2\right)-\hat{\beta}_{n} \hat{b} \sin \left(\hat{\beta}_{n} / 2\right)\right]\left[\sin \left(\hat{\beta}_{n} / 2\right)+\hat{\beta}_{n} \hat{b} \cos \left(\hat{\beta}_{n} / 2\right)\right]=0
$$

from which we may separate $\hat{\beta}_{n}$ into two sets. For $n=1,3,5, \cdots, \hat{\beta}_{n}$ are the roots of

$$
\cos \left(\hat{\beta}_{n} / 2\right)-\hat{\beta}_{n} \hat{b} \sin \left(\hat{\beta}_{n} / 2\right)=0
$$

while for $n=2,4,6, \cdots, \hat{\beta}_{n}$ are the roots of

$$
\sin \left(\hat{\beta}_{n} / 2\right)+\hat{\beta}_{n} \hat{b} \cos \left(\hat{\beta}_{n} / 2\right)=0
$$

Meanwhile, the numerator of $\hat{A}_{n}$, given in Eq. (68), can be written as

$$
8 \sin \left(\hat{\beta}_{n} / 2\right)\left[\sin \left(\hat{\beta}_{n} / 2\right)+\hat{\beta}_{n} \hat{b} \cos \left(\hat{\beta}_{n} / 2\right)\right]
$$

which by Eq. (75) is zero for even values of $n$. Therefore, in the special case of equal slip lengths on the two walls, $\hat{\beta}_{2,4,6, \ldots}$ can be ignored, and the characteristic equation thereby reduces to Eq. (74). With equal slip lengths, the flow is symmetrical about the mid-plane of the channel, and the solution can be expressed in a simpler form if the origin of the $y$-axis is set at the mid-point between the two walls. Let us introduce the following revised normalized quantities (distinguished by overhead tildes), where half the channel height, $h / 2$, is used as the length scale for the normalization: $\tilde{u}=4 \hat{u}, \tilde{y}=2 \hat{y}-1, \tilde{b}=2 \hat{b}, \tilde{\beta}_{n}=\hat{\beta}_{n} / 2$, and $\tilde{t}=4 \hat{t}$. In terms of these revised normalized quantities, we may express

$$
\tilde{u}(\tilde{y}, \tilde{t})=\frac{1}{2}\left(1-\tilde{y}^{2}+2 \tilde{b}\right)-4 \sum_{n=1}^{\infty} \frac{\sin \left(\tilde{\beta}_{n}\right) \cos \left(\tilde{\beta}_{n} \tilde{y}\right)}{\tilde{\beta}_{n}^{2}\left[2 \tilde{\beta}_{n}+\sin \left(2 \tilde{\beta}_{n}\right)\right]} e^{-\tilde{\beta}_{n}^{2} \tilde{t}}
$$

where $\tilde{\beta}_{n}$ are the roots of

$$
\cos \left(\tilde{\beta}_{n}\right)-\tilde{\beta}_{n} \tilde{b} \sin \left(\tilde{\beta}_{n}\right)=0 .
$$

This transient velocity profile, which is for equal slip lengths, is the same as the one given by Kaoullas and Georgiou [14] in their static slip case, and also the one by Avramenko et al. [11], albeit in different forms. 

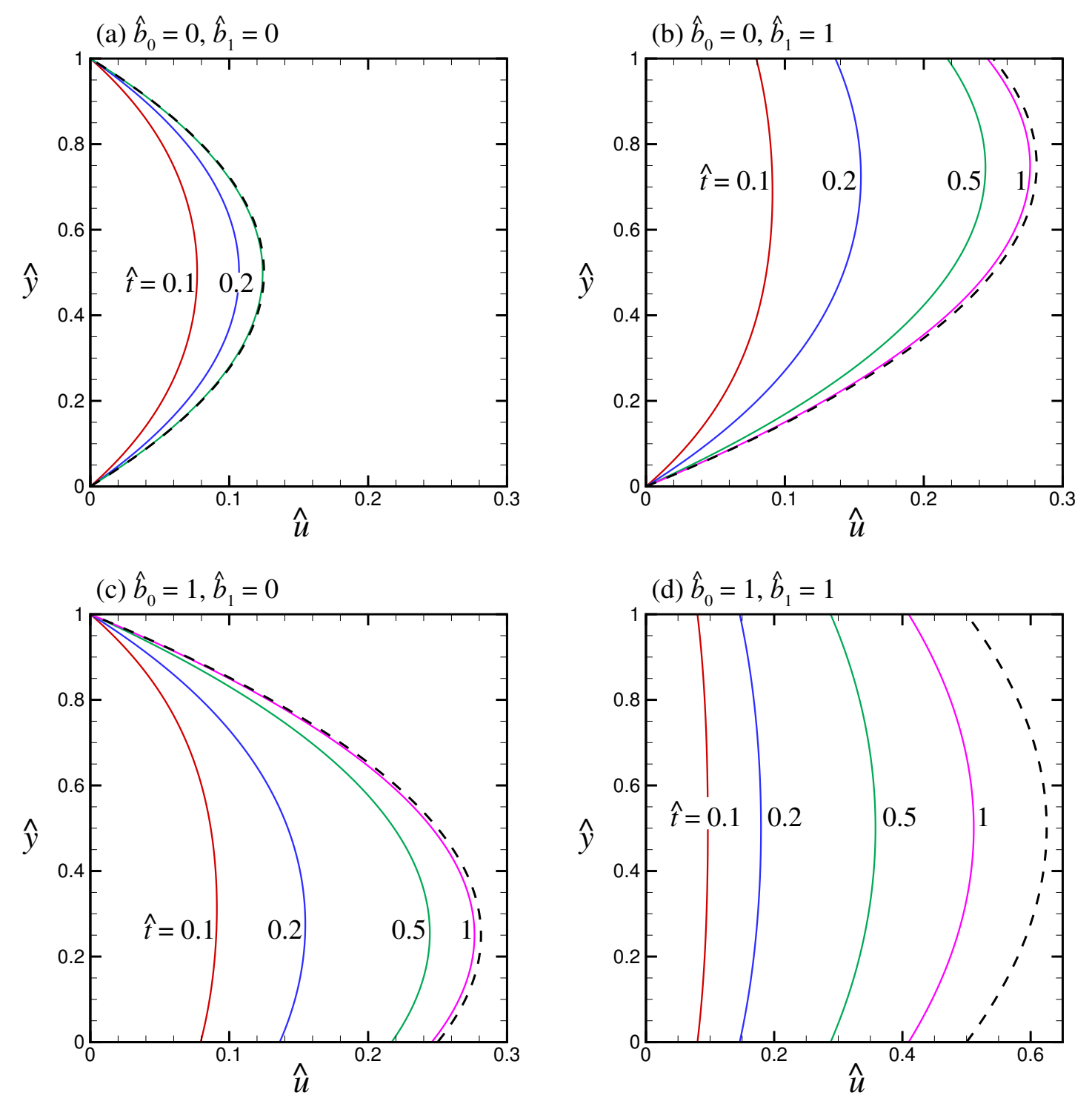

Figure 9: For starting Poiseuille flow through a parallel-plate channel, the velocity profile $\hat{u}(\hat{y})$ as a function of $\hat{t}$, for $(\mathrm{a}) \hat{b}_{0}=0, \hat{b}_{1}=0$; (b) $\hat{b}_{0}=0, \hat{b}_{1}=1 ;$ (c) $\hat{b}_{0}=1, \hat{b}_{1}=0 ;$ (d) $\hat{b}_{0}=1, \hat{b}_{1}=1$. The dashed lines denote the steady-state velocity profiles. 
We show in Fig. 9 the velocity profile $\hat{u}(\hat{y})$ as a function of time for four sets of values

of the slip lengths. Again, we see that without any boundary $\operatorname{slip}\left(\hat{b}_{0}=\hat{b}_{1}=0\right)$, the steady state is practically attained within a short duration, $\hat{t}<0.5$. Boundary slip leads to a longer transient period, basically because of a longer time for the velocity near a slipping boundary to reach the steady state. It is evident from the figure that the boundary slip is to reduce the near-wall velocity gradient. Hence, as has been remarked above, the slower decaying transient in the presence of boundary slip is essentially due to a lower rate of momentum diffusion across the boundary where the slip occurs.

\section{Poiseuille flow through a rectangular channel}

Our next problem is to consider starting pressure-driven flow through a rectangular channel of height $2 h$ and width $2 a$, with a uniform slip length $b$ on all the walls; see Fig. 10 . The problem is as follows:

$$
\frac{\partial u}{\partial t}=-\frac{1}{\rho} \frac{\partial p}{\partial z}+\nu\left(\frac{\partial^{2} u}{\partial x^{2}}+\frac{\partial^{2} u}{\partial y^{2}}\right) \quad \text { in }-a \leq x \leq a,-h \leq y \leq h,
$$

where $u(x, y, t)$ is the velocity in the $z$-direction, and the axial pressure gradient is

$$
-\frac{\partial p}{\partial z}=\left\{\begin{array}{c}
K, \quad t>0 \\
0, \quad t<0
\end{array}\right.
$$

in which $K$ is the suddenly imposed pressure gradient. The boundary conditions are

$$
\begin{array}{ll}
u=\mp b \frac{\partial u}{\partial y} & \text { at } y= \pm h, \\
u=\mp b \frac{\partial u}{\partial x} & \text { at } x= \pm a .
\end{array}
$$

Start-up slip flow through a rectangular channel has also been investigated by Avramenko et al. [12]. They considered a pressure gradient that may vary with time in a power-law manner. Their solutions, in terms of hypergeometric functions and their integrals, are rather complicated in form. Our problem formulated above for the sudden imposition of a constant pressure gradient can be solved in terms of elementary functions. 


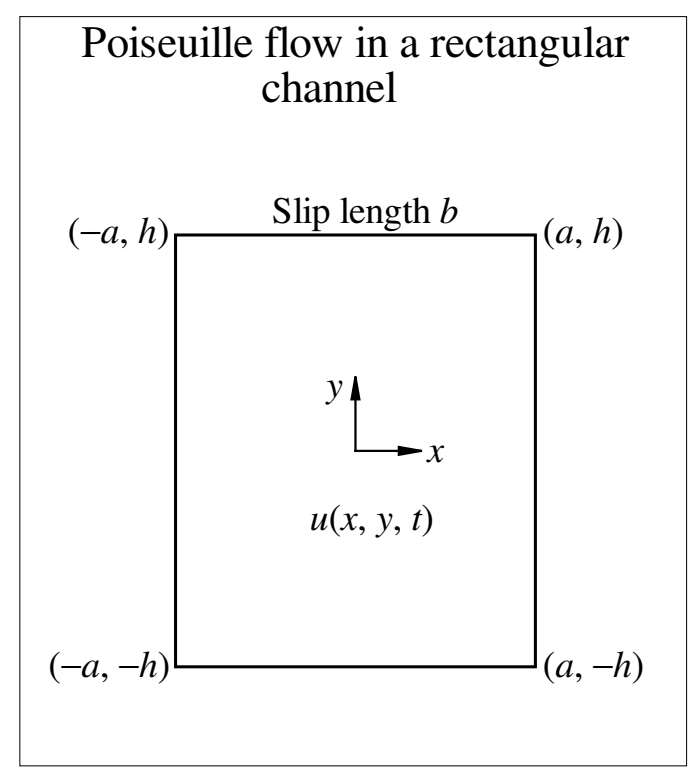

Figure 10: Starting Poiseuille flow through a rectangular channel, where the flow is perpendicular to the plane of paper.

Again, we first decompose the velocity into steady and transient components

$$
u(x, y, t)=u_{\mathrm{S}}(x, y)-u_{\mathrm{T}}(x, y, t)
$$

where $u_{\mathrm{T}}$ matches $u_{\mathrm{S}}$ at $t=0$, and decays to zero for large times. Using eigenfunction expansions, the steady-state solution is found as below:

$$
u_{\mathrm{S}}(x, y)=\frac{K h^{2}}{2 \mu}\left(1-\frac{y^{2}}{h^{2}}+\frac{2 b}{h}\right)+\sum_{n=1}^{\infty} A_{n} \cosh \left(\alpha_{n} x\right) \cos \left(\alpha_{n} y\right)
$$

where $\alpha_{n}$ are the positive roots of

$$
\cos \left(\alpha_{n} h\right)-\alpha_{n} b \sin \left(\alpha_{n} h\right)=0
$$

and

$$
A_{n}=-\frac{4 K \sin \left(\alpha_{n} h\right)}{\mu \alpha_{n}^{2}\left[\cosh \left(\alpha_{n} a\right)+\alpha_{n} b \sinh \left(\alpha_{n} a\right)\right]\left[2 \alpha_{n} h+\sin \left(2 \alpha_{n} h\right)\right]} .
$$

Also using eigenfunction expansions, the transient solution is expressible by

$$
u_{\mathrm{T}}(x, y, t)=\sum_{n=1}^{\infty} \sum_{m=1}^{\infty} B_{m n} \cos \left(\gamma_{m} x\right) \cos \left(\alpha_{n} y\right) e^{-\nu \beta_{m n}^{2} t}
$$

where

$$
\beta_{m n}^{2}=\gamma_{m}^{2}+\alpha_{n}^{2}
$$


and $\gamma_{m}$ are the positive roots of

$$
\cos \left(\gamma_{m} a\right)-\gamma_{m} b \sin \left(\gamma_{m} a\right)=0
$$

The coefficients $B_{m n}$ are determined by the initial condition $u_{\mathrm{T}}(t=0)=u_{\mathrm{S}}$. Using orthogonality of the eigenfunctions, we may obtain after some algebra

$$
B_{m n}=\frac{16 K \sin \left(\gamma_{m} a\right) \sin \left(\alpha_{n} h\right)}{\mu \beta_{m n}^{2}\left[2 \gamma_{m} a+\sin \left(2 \gamma_{m} a\right)\right]\left[2 \alpha_{n} h+\sin \left(2 \alpha_{n} h\right)\right]}
$$

In terms of normalized variables: $\left(\hat{u}, \hat{A}_{n}, \hat{B}_{m n}\right)=\left(u, A_{n}, B_{m n}\right) /\left(K h^{2} / \mu\right),(\hat{x}, \hat{y}, \hat{a}, \hat{b})=$ $(x, y, a, b) / h,\left(\hat{\alpha}_{n}, \hat{\gamma}_{m}, \hat{\beta}_{m n}\right)=\left(\alpha_{n}, \gamma_{m}, \beta_{m n}\right) h$, and $\hat{t}=t\left(\nu / h^{2}\right)$, the velocity for the present problem is

$$
\begin{aligned}
\hat{u}(\hat{x}, \hat{y}, \hat{t})= & \frac{1}{2}\left(1-\hat{y}^{2}+2 \hat{b}\right)+\sum_{n=1}^{\infty} \hat{A}_{n} \cosh \left(\hat{\alpha}_{n} \hat{x}\right) \cos \left(\hat{\alpha}_{n} \hat{y}\right) \\
& -\sum_{n=1}^{\infty} \sum_{m=1}^{\infty} \hat{B}_{m n} \cos \left(\hat{\gamma}_{m} \hat{x}\right) \cos \left(\hat{\alpha}_{n} \hat{y}\right) e^{-\hat{\beta}_{m n}^{2} \hat{t}}
\end{aligned}
$$

where

$$
\begin{gathered}
\hat{A}_{n}=-\frac{4 \sin \left(\hat{\alpha}_{n}\right)}{\hat{\alpha}_{n}^{2}\left[\cosh \left(\hat{\alpha}_{n} \hat{a}\right)+\hat{\alpha}_{n} \hat{b} \sinh \left(\hat{\alpha}_{n} \hat{a}\right)\right]\left[2 \hat{\alpha}_{n}+\sin \left(2 \hat{\alpha}_{n}\right)\right]}, \\
\hat{B}_{m n}=\frac{16 \sin \left(\hat{\gamma}_{m} \hat{a}\right) \sin \left(\hat{\alpha}_{n}\right)}{\hat{\beta}_{m n}^{2}\left[2 \hat{\gamma}_{m} \hat{a}+\sin \left(2 \hat{\gamma}_{m} \hat{a}\right)\right]\left[2 \hat{\alpha}_{n}+\sin \left(2 \hat{\alpha}_{n}\right)\right]}, \\
\hat{\beta}_{m n}^{2}=\hat{\gamma}_{m}^{2}+\hat{\alpha}_{n}^{2}
\end{gathered}
$$

and $\hat{\gamma}_{m}$ and $\hat{\alpha}_{n}$ are found from the following characteristic equations

$$
\begin{gathered}
\cos \left(\hat{\gamma}_{m} \hat{a}\right)-\hat{\gamma}_{m} \hat{b} \sin \left(\hat{\gamma}_{m} \hat{a}\right)=0 \\
\cos \left(\hat{\alpha}_{n}\right)-\hat{\alpha}_{n} \hat{b} \sin \left(\hat{\alpha}_{n}\right)=0 .
\end{gathered}
$$

For future reference, we provide in Table A3 the first five values of $\hat{\gamma}_{m}$ for some values of $\hat{a}$ and $\hat{b}$. The rate of flow through the channel is

$$
\begin{aligned}
\hat{Q}(\hat{t})= & \int_{-\hat{a}}^{\hat{a}} \int_{-1}^{1} \hat{u} \mathrm{~d} \hat{y} \mathrm{~d} \hat{x} \\
= & \frac{4 \hat{a}}{3}(1+3 \hat{b})+4 \sum_{n=1}^{\infty} \frac{\hat{A}_{n}}{\hat{\alpha}_{n}^{2}} \sinh \left(\hat{\alpha}_{n} \hat{a}\right) \sin \left(\hat{\alpha}_{n}\right) \\
& -4 \sum_{n=1}^{\infty} \sum_{m=1}^{\infty} \frac{\hat{B}_{m n}}{\hat{\gamma}_{m} \hat{\alpha}_{n}} \sin \left(\hat{\gamma}_{m} \hat{a}\right) \sin \left(\hat{\alpha}_{n}\right) e^{-\hat{\beta}_{m n}^{2} \hat{t}}
\end{aligned}
$$




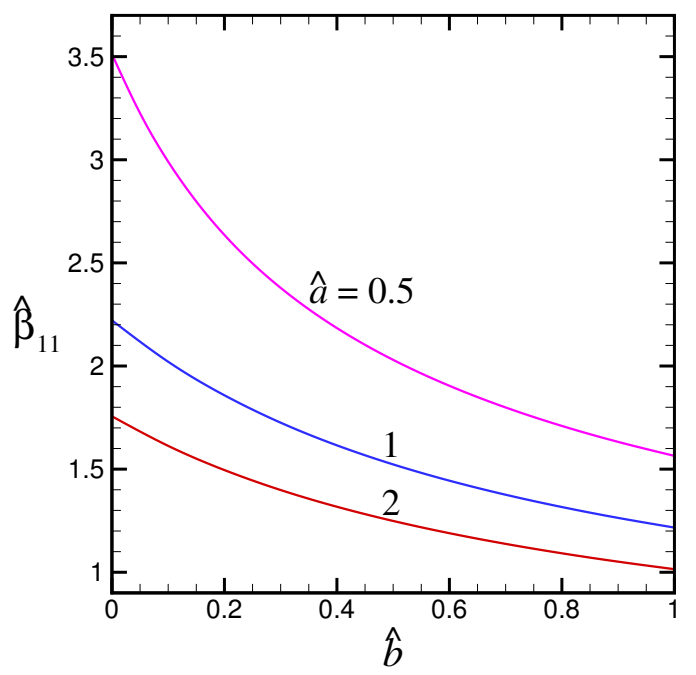

Figure 11: For starting Poiseuille flow through a rectangular channel, the leading eigenvalue $\hat{\beta}_{11}$ as a function of the slip length $\hat{b}$ and the channel width $\hat{a}$.
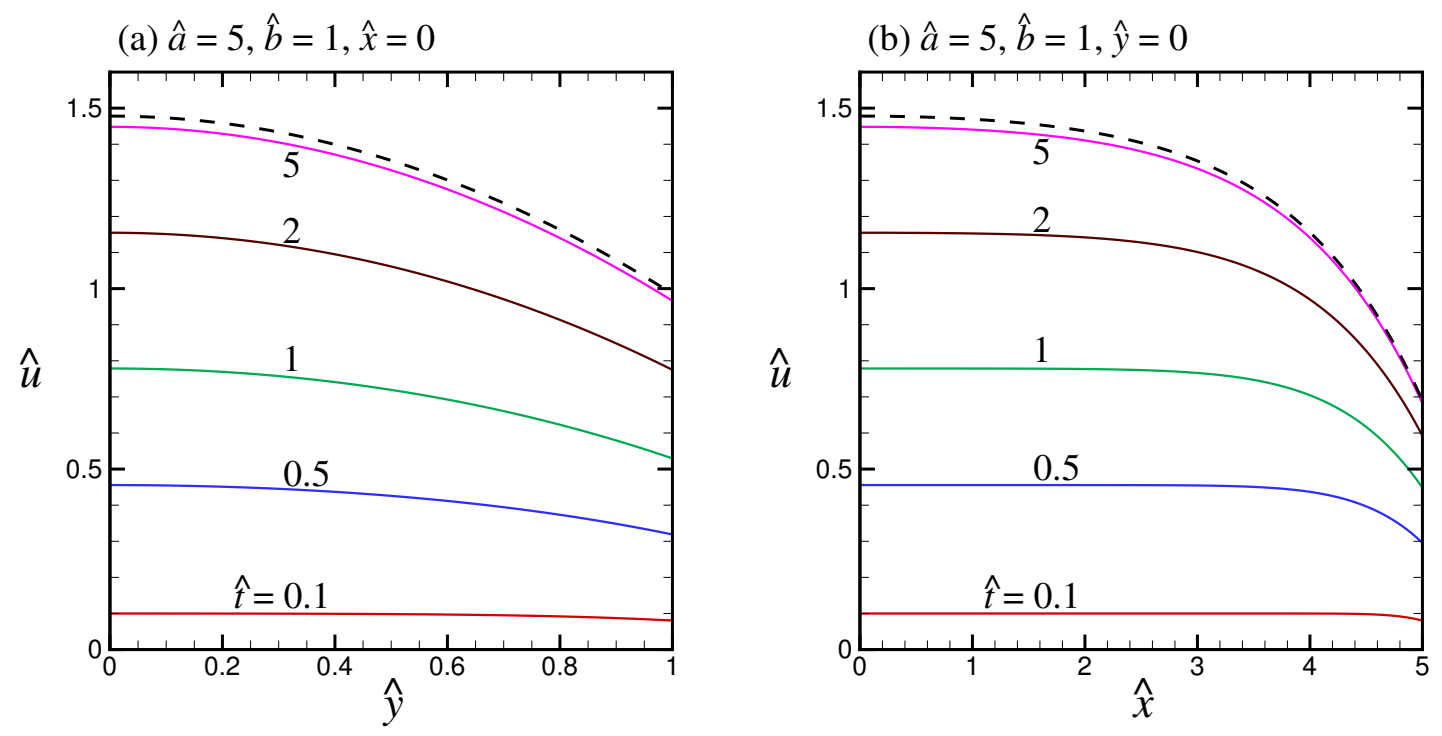

Figure 12: For starting Poiseuille flow through a rectangular channel of aspect ratio $\hat{a}=5$ and slip length $\hat{b}=1$, the velocity profiles at various times in the two middle cross sections: (a) $\hat{u}(0, \hat{y}) ;(\mathrm{b}) \hat{u}(\hat{x}, 0)$. The dashed lines denote the steady-state velocity profiles. 
We show in Fig. 11 the leading eigenvalue $\hat{\beta}_{11}$ as a function of the slip length $\hat{b}$, for channel width $a=0.5,1,2$. Again, it is seen that the eigenvalue decreases as the slip length increases, implying a longer transient time for a more slipping boundary. It is also seen that a smaller eigenvalue results from a larger channel width, implying that it takes a longer time for the transient to die out in a wider channel, which is expected. We further show in Fig. 12 the developing profiles in the two middle cross sections $\hat{x}=0$ and $\hat{y}=0$ for $\hat{a}=5$ and $\hat{b}=1$. As has been remarked by Avramenko et al. [12], the velocity slip is larger along the longer walls (i.e., $\hat{y}= \pm 1$ ). This is essentially a result of a larger velocity gradient near these two walls. Despite a large aspect ratio (the two vertical walls are 5 times farther apart than the two horizontal walls in this example), the time taken to reach the steady state is nearly the same in either direction.

\section{Poiseuille flow through a circular channel}

We next consider starting pressure-driven flow through a circular channel of radius $R$, with a uniform slip length $b$ on the wall; see Fig. 13. The problem is as follows:

$$
\frac{\partial u}{\partial t}=-\frac{1}{\rho} \frac{\partial p}{\partial z}+\frac{\nu}{r} \frac{\partial}{\partial r}\left(r \frac{\partial u}{\partial r}\right) \quad \text { in } 0 \leq r \leq R
$$

where $u(r, t)$ is the velocity in the $z$-direction, and the axial pressure gradient is

$$
-\frac{\partial p}{\partial z}=\left\{\begin{array}{c}
K, \quad t>0 \\
0, \quad t<0
\end{array}\right.
$$

in which $K$ is the suddenly applied pressure gradient. The boundary conditions are

$$
\begin{array}{cl}
u=\text { finite } & \text { at } r=0, \\
u=-b \frac{\partial u}{\partial r} & \text { at } r=R .
\end{array}
$$

The same problem has also been solved by Avramenko et al. [11] using Laplace transform. Their method of analysis, however, results in a solution containing hypergeometric functions, which unnecessarily complicate the expression of the solution. We shall deduce below a more compact solution that reads the same as the one obtained by Kaoullas and Georgiou [14] without the dynamic slip. 


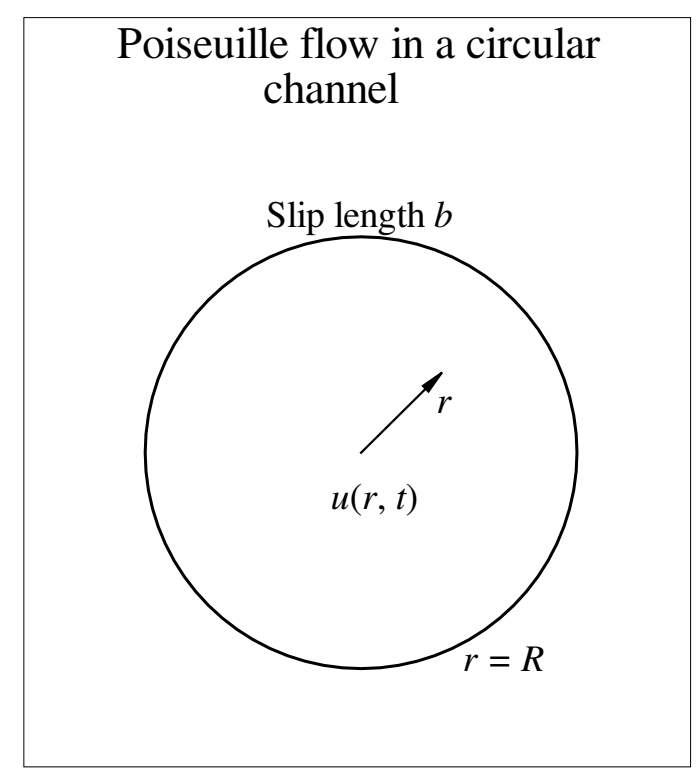

Figure 13: Starting Poiseuille flow through a circular channel, where the flow is perpendicular to the plane of paper.

As in the preceding problems, we decompose the velocity into steady and transient components:

$$
u(r, t)=u_{\mathrm{S}}(r)-u_{\mathrm{T}}(r, t),
$$

where the transient velocity is equal to the steady velocity at $t=0$, and decays to zero as $t \rightarrow \infty$. The steady-state velocity is readily found to be

$$
u_{\mathrm{S}}(r)=\frac{K R^{2}}{4 \mu}\left(1-\frac{r^{2}}{R^{2}}+\frac{2 b}{R}\right),
$$

while the transient velocity satisfying the momentum equation (without the forcing term) and boundary conditions is expressible by

$$
u_{\mathrm{T}}(r, t)=\sum_{n=1}^{\infty} A_{n} J_{0}\left(\beta_{n} r\right) e^{-\nu \beta_{n}^{2} t}
$$

where $\beta_{n}$ are the positive roots of

$$
J_{0}\left(\beta_{n} R\right)-\beta_{n} b J_{1}\left(\beta_{n} R\right)=0
$$

and $J_{k}$ is the Bessel function of the first kind of order $k$. Using the initial condition $u_{\mathrm{T}}(t=$ $0)=u_{\mathrm{s}}$ and orthogonality of the eigenfunctions (with $r$ being the weighting function), we 
may determine the coefficients as follows:

$$
A_{n}=\frac{2 K}{\mu \beta_{n}^{3} R\left(1+\beta_{n}^{2} b^{2}\right) J_{1}\left(\beta_{n} R\right)} .
$$

The transient velocity deduced here agrees with the one previously found by Kaoullas and Georgiou [14] in their case of zero dynamic slip.

In terms of normalized variables: $\left(\hat{u}, \hat{A}_{n}\right)=\left(u, A_{n}\right) /\left(K R^{2} / \mu\right),(\hat{r}, \hat{b})=(r, b) / R, \hat{\beta}_{n}=\beta_{n} R$, and $\hat{t}=t\left(\nu / R^{2}\right)$, the velocity for the present problem is

$$
\hat{u}(\hat{r}, \hat{t})=\frac{1}{4}\left(1-\hat{r}^{2}+2 \hat{b}\right)-\sum_{n=1}^{\infty} \hat{A}_{n} J_{0}\left(\hat{\beta}_{n} \hat{r}\right) e^{-\hat{\beta}_{n}^{2} \hat{t}},
$$

where

$$
\hat{A}_{n}=\frac{2}{\hat{\beta}_{n}^{3}\left(1+\hat{\beta}_{n}^{2} \hat{b}^{2}\right) J_{1}\left(\hat{\beta}_{n}\right)},
$$

and $\hat{\beta}_{n}$ are the positive roots of

$$
J_{0}\left(\hat{\beta}_{n}\right)-\hat{\beta}_{n} \hat{b} J_{1}\left(\hat{\beta}_{n}\right)=0 .
$$

For future reference, we provide in Table A4 the first five values of $\hat{\beta}_{n}$ for some values of $\hat{b}$. The rate of flow through the channel is

$$
\hat{Q}(\hat{t})=2 \pi \int_{0}^{1} \hat{r} \hat{u} \mathrm{~d} \hat{r}=\frac{\pi}{8}(1+4 \hat{b})-2 \pi \sum_{n=1}^{\infty} \frac{\hat{A}_{n}}{\hat{\beta}_{n}} J_{1}\left(\hat{\beta}_{n}\right) e^{-\hat{\beta}_{n}^{2} \hat{t}} .
$$

For a no-slip wall, $\hat{b}=0$, the eigenvalues $\hat{\beta}_{n}$ are the zeros of $J_{0}$, and $\hat{A}_{n}=2 /\left[\hat{\beta}_{n}^{3} J_{1}\left(\hat{\beta}_{n}\right)\right]$. Equations (107) and (110) can then be written as

$$
\hat{u}_{\text {no-slip }}=\frac{1}{4}\left(1-\hat{r}^{2}\right)-2 \sum_{n=1}^{\infty} \frac{J_{0}\left(\hat{\beta}_{n} \hat{r}\right)}{\hat{\beta}_{n}^{3} J_{1}\left(\hat{\beta}_{n}\right)} e^{-\hat{\beta}_{n}^{2} \hat{t}},
$$

and

$$
\hat{Q}_{\text {no-slip }}=\frac{\pi}{8}-4 \pi \sum_{n=1}^{\infty} \frac{e^{-\hat{\beta}_{n}^{2} \hat{t}}}{\hat{\beta}_{n}^{4}} .
$$

Equation (111) is known as the Szymanski [21] solution; see White [19].

The leading eigenvalue $\hat{\beta}_{1}$ is shown in Fig. 14 as a function of the slip length $\hat{b}$. The curve shows a decreasing effect of the slip length on the eigenvalue, in a manner similar to those already seen in Figs. 4 and 11. 


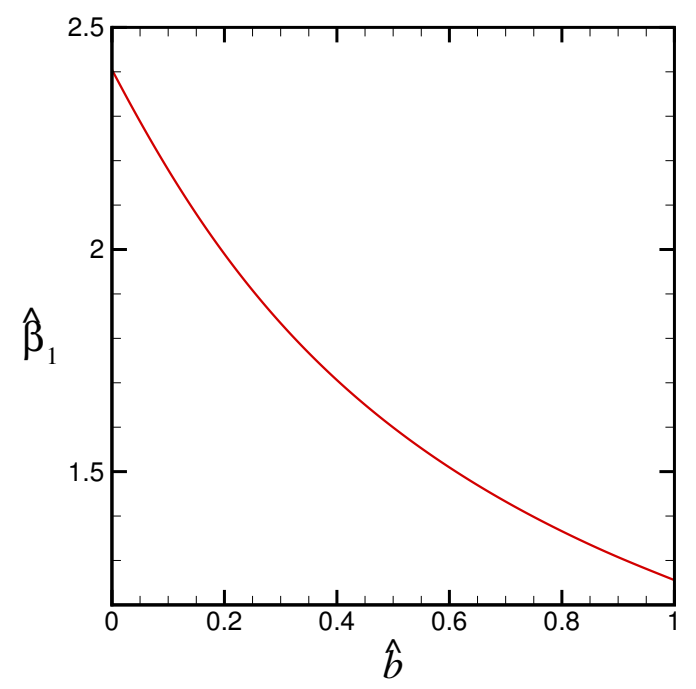

Figure 14: For starting Poiseuille flow through a circular channel, the leading eigenvalue $\hat{\beta}_{1}$ as a function of the slip length $\hat{b}$.
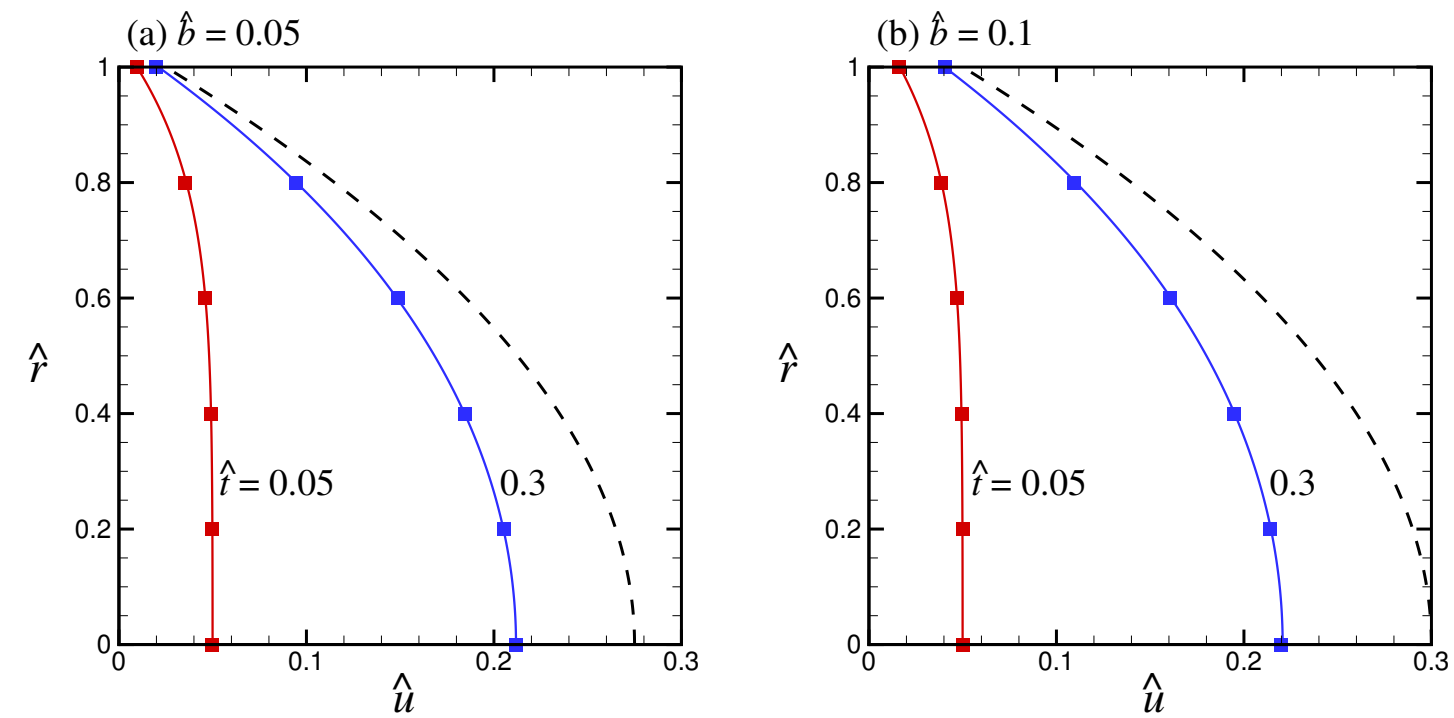

Figure 15: For starting Poiseuille flow through a circular channel, the velocity profile $\hat{u}(\hat{r})$ at $\hat{t}=0.05,0.3$, for $(\mathrm{a}) \hat{b}=0.05$; (b) $\hat{b}=0.1$. The dashed lines denote the steady-state velocity profiles. The square symbols are results computed by Avramenko et al. [11]. 
To confirm that our solution is indeed the same as the one deduced by Avramenko et al. [11], we show in Fig. 15 the velocity profile $\hat{u}(\hat{r})$ at $\hat{t}=0.05,0.3$ for $\hat{b}=0.05,0.1$, where we show also results (the squares) computed by Avramenko et al. [11]. This figure clearly indicates agreement of results obtained from their and our solutions. Their transient velocity, which is an infinite series containing a hypergeometric function, can be simplified to the one expressed above.

\section{Poiseuille flow through a concentric annulus}

We finally consider starting pressure-driven flow through the annulus between an inner cylinder of radius $R_{0}$ and an outer concentric cylinder of radius $R_{1}$; see Fig. 16. The slip lengths on the inner and outer walls are $b_{0}$ and $b_{1}$, respectively. The problem, which is important in viscometry, is as follows:

$$
\frac{\partial u}{\partial t}=-\frac{1}{\rho} \frac{\partial p}{\partial z}+\frac{\nu}{r} \frac{\partial}{\partial r}\left(r \frac{\partial u}{\partial r}\right) \quad \text { in } R_{0} \leq r \leq R_{1},
$$

where $u(r, t)$ is the axial velocity in the $z$-direction, and the axial pressure gradient is

$$
-\frac{\partial p}{\partial z}=\left\{\begin{array}{cc}
K, & t>0 \\
0, & t<0
\end{array},\right.
$$

in which $K$ is the pressure gradient suddenly imposed along the channel. The boundary conditions are

$$
\begin{gathered}
u=b_{0} \frac{\partial u}{\partial r} \quad \text { at } r=R_{0}, \\
u=-b_{1} \frac{\partial u}{\partial r} \quad \text { at } r=R_{1} .
\end{gathered}
$$

As in the preceding problems, we decompose the velocity into steady and transient parts:

$$
u(r, t)=u_{\mathrm{S}}(r)-u_{\mathrm{T}}(r, t),
$$

where $u_{\mathrm{T}}$ is equal to $u_{\mathrm{S}}$ at $t=0$, and decays to zero as $t \rightarrow \infty$. The steady-state velocity is readily found to be

$$
u_{\mathrm{S}}(r)=\frac{K}{4 \mu}\left[-r^{2}+c_{1} \ln \left(\frac{r}{R_{1}}\right)+c_{2}\right],
$$




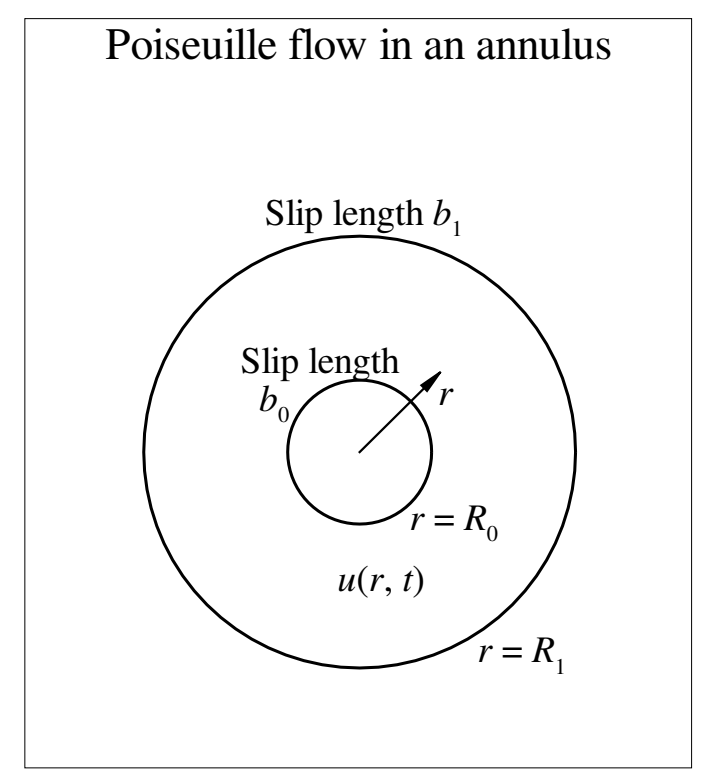

Figure 16: Starting Poiseuille flow through a concentric annulus, where the flow is perpendicular to the plane of paper.

where

$$
\begin{gathered}
c_{1}=\frac{R_{1}^{2}-R_{0}^{2}+2 R_{1} b_{1}+2 R_{0} b_{0}}{\ln \left(R_{1} / R_{0}\right)+b_{1} / R_{1}+b_{0} / R_{0}}, \\
c_{2}=\frac{\left(R_{0}^{2}-2 R_{0} b_{0}\right)\left(b_{1} / R_{1}\right)+\left(R_{1}^{2}+2 R_{1} b_{1}\right)\left[\ln \left(R_{1} / R_{0}\right)+b_{0} / R_{0}\right]}{\ln \left(R_{1} / R_{0}\right)+b_{1} / R_{1}+b_{0} / R_{0}} .
\end{gathered}
$$

Using eigenfunction expansions, the transient velocity that satisfies the momentum equation (without the forcing term) and boundary conditions is expressible by

$$
u_{\mathrm{T}}(r, t)=\sum_{n=1}^{\infty} A_{n}\left[J_{0}\left(\beta_{n} r\right)+\lambda_{n} Y_{0}\left(\beta_{n} r\right)\right] e^{-\nu \beta_{n}^{2} t}
$$

where

$$
\lambda_{n}=-\frac{J_{0}\left(\beta_{n} R_{0}\right)+\beta_{n} b_{0} J_{1}\left(\beta_{n} R_{0}\right)}{Y_{0}\left(\beta_{n} R_{0}\right)+\beta_{n} b_{0} Y_{1}\left(\beta_{n} R_{0}\right)},
$$

and $\beta_{n}$ are the positive roots of the following characteristic equation

$$
\begin{aligned}
& {\left[J_{0}\left(\beta_{n} R_{0}\right)+\beta_{n} b_{0} J_{1}\left(\beta_{n} R_{0}\right)\right]\left[Y_{0}\left(\beta_{n} R_{1}\right)-\beta_{n} b_{1} Y_{1}\left(\beta_{n} R_{1}\right)\right]} \\
& -\left[J_{0}\left(\beta_{n} R_{1}\right)-\beta_{n} b_{1} J_{1}\left(\beta_{n} R_{1}\right)\right]\left[Y_{0}\left(\beta_{n} R_{0}\right)+\beta_{n} b_{0} Y_{1}\left(\beta_{n} R_{0}\right)\right]=0 .
\end{aligned}
$$

In the above equations, $J_{k}$ and $Y_{k}$ are respectively the Bessel functions of the first and the second kinds of order $k$. 
The coefficients $A_{n}$ are found from the initial condition $u_{\mathrm{T}}(t=0)=u_{\mathrm{S}}$. Using orthogonality (with $r$ being the weighting function) of the eigenfunctions, we may obtain after some algebra:

$$
A_{n}=\frac{2 K}{\mu \beta_{n}^{3} R_{1}}\left(\frac{D_{n}}{E_{n}}\right)
$$

where

$$
\begin{aligned}
D_{n}= & \frac{J_{1}\left(\beta_{n} R_{1}\right) Y_{0}\left(\beta_{n} R_{1}\right)-J_{0}\left(\beta_{n} R_{1}\right) Y_{1}\left(\beta_{n} R_{1}\right)}{Y_{0}\left(\beta_{n} R_{1}\right)-\beta_{n} b_{1} Y_{1}\left(\beta_{n} R_{1}\right)} \\
& -\left(\frac{R_{0}}{R_{1}}\right) \frac{J_{1}\left(\beta_{n} R_{0}\right) Y_{0}\left(\beta_{n} R_{0}\right)-J_{0}\left(\beta_{n} R_{0}\right) Y_{1}\left(\beta_{n} R_{0}\right)}{Y_{0}\left(\beta_{n} R_{0}\right)+\beta_{n} b_{0} Y_{1}\left(\beta_{n} R_{0}\right)}, \\
E_{n}= & \left(1+\beta_{n}^{2} b_{1}^{2}\right)\left[\frac{J_{1}\left(\beta_{n} R_{1}\right) Y_{0}\left(\beta_{n} R_{1}\right)-J_{0}\left(\beta_{n} R_{1}\right) Y_{1}\left(\beta_{n} R_{1}\right)}{Y_{0}\left(\beta_{n} R_{1}\right)-\beta_{n} b_{1} Y_{1}\left(\beta_{n} R_{1}\right)}\right]^{2} \\
& -\left(1+\beta_{n}^{2} b_{0}^{2}\right)\left(\frac{R_{0}}{R_{1}}\right)^{2}\left[\frac{J_{1}\left(\beta_{n} R_{0}\right) Y_{0}\left(\beta_{n} R_{0}\right)-J_{0}\left(\beta_{n} R_{0}\right) Y_{1}\left(\beta_{n} R_{0}\right)}{Y_{0}\left(\beta_{n} R_{0}\right)+\beta_{n} b_{0} Y_{1}\left(\beta_{n} R_{0}\right)}\right]^{2} .
\end{aligned}
$$

Introducing the following normalized quantities: $\left(\hat{u}, \hat{A}_{n}\right)=\left(u, A_{n}\right) /\left(K R_{1}^{2} / \mu\right),\left(\hat{r}, \hat{b}, \hat{R}_{0}\right)=$ $\left(r, b, R_{0}\right) / R_{1}, \hat{\beta}_{n}=\beta_{n} R_{1}$, and $\hat{t}=t\left(\nu / R_{1}^{2}\right)$, the velocity for the present problem in dimensionless form is

$$
\hat{u}(\hat{r}, \hat{t})=\frac{1}{4}\left[-\hat{r}^{2}+\hat{c}_{1} \ln (\hat{r})+\hat{c}_{2}\right]-2 \sum_{n=1}^{\infty} \frac{D_{n}}{\hat{\beta}_{n}^{3} E_{n}}\left[J_{0}\left(\hat{\beta}_{n} \hat{r}\right)+\lambda_{n} Y_{0}\left(\hat{\beta}_{n} \hat{r}\right)\right] e^{-\hat{\beta}_{n}^{2} \hat{t}}
$$

where

$$
\begin{gathered}
\hat{c}_{1}=\frac{1-\hat{R}_{0}^{2}+2 \hat{b}_{1}+2 \hat{R}_{0} \hat{b}_{0}}{\ln \left(1 / \hat{R}_{0}\right)+\hat{b}_{1}+\hat{b}_{0} / \hat{R}_{0}}, \\
\hat{c}_{2}=\frac{\left(\hat{R}_{0}^{2}-2 \hat{R}_{0} \hat{b}_{0}\right) \hat{b}_{1}+\left(1+2 \hat{b}_{1}\right)\left[\ln \left(1 / \hat{R}_{0}\right)+\hat{b}_{0} / \hat{R}_{0}\right]}{\ln \left(1 / \hat{R}_{0}\right)+\hat{b}_{1}+\hat{b}_{0} / \hat{R}_{0}}
\end{gathered}
$$

and $\hat{\beta}_{n}$ are the positive roots of

$$
\begin{aligned}
& {\left[J_{0}\left(\hat{\beta}_{n} \hat{R}_{0}\right)+\hat{\beta}_{n} \hat{b}_{0} J_{1}\left(\hat{\beta}_{n} \hat{R}_{0}\right)\right]\left[Y_{0}\left(\hat{\beta}_{n}\right)-\hat{\beta}_{n} \hat{b}_{1} Y_{1}\left(\hat{\beta}_{n}\right)\right]} \\
& -\left[J_{0}\left(\hat{\beta}_{n}\right)-\hat{\beta}_{n} \hat{b}_{1} J_{1}\left(\hat{\beta}_{n}\right)\right]\left[Y_{0}\left(\hat{\beta}_{n} \hat{R}_{0}\right)+\hat{\beta}_{n} \hat{b}_{0} Y_{1}\left(\hat{\beta}_{n} \hat{R}_{0}\right)\right]=0 .
\end{aligned}
$$

The coefficients $\lambda_{n}, D_{n}$ and $E_{n}$ in terms of the normalized parameters are

$$
\lambda_{n}=-\frac{J_{0}\left(\hat{\beta}_{n} \hat{R}_{0}\right)+\hat{\beta}_{n} \hat{b}_{0} J_{1}\left(\hat{\beta}_{n} \hat{R}_{0}\right)}{Y_{0}\left(\hat{\beta}_{n} \hat{R}_{0}\right)+\hat{\beta}_{n} \hat{b}_{0} Y_{1}\left(\hat{\beta}_{n} \hat{R}_{0}\right)}
$$




$$
\begin{aligned}
D_{n}= & \frac{J_{1}\left(\hat{\beta}_{n}\right) Y_{0}\left(\hat{\beta}_{n}\right)-J_{0}\left(\hat{\beta}_{n}\right) Y_{1}\left(\hat{\beta}_{n}\right)}{Y_{0}\left(\hat{\beta}_{n}\right)-\hat{\beta}_{n} \hat{b}_{1} Y_{1}\left(\hat{\beta}_{n}\right)} \\
& -\hat{R}_{0} \frac{J_{1}\left(\hat{\beta}_{n} \hat{R}_{0}\right) Y_{0}\left(\hat{\beta}_{n} \hat{R}_{0}\right)-J_{0}\left(\hat{\beta}_{n} \hat{R}_{0}\right) Y_{1}\left(\hat{\beta}_{n} \hat{R}_{0}\right)}{Y_{0}\left(\hat{\beta}_{n} \hat{R}_{0}\right)+\hat{\beta}_{n} \hat{b}_{0} Y_{1}\left(\hat{\beta}_{n} \hat{R}_{0}\right)}, \\
E_{n}= & \left(1+\hat{\beta}_{n}^{2} \hat{b}_{1}^{2}\right)\left[\frac{J_{1}\left(\hat{\beta}_{n}\right) Y_{0}\left(\hat{\beta}_{n}\right)-J_{0}\left(\hat{\beta}_{n}\right) Y_{1}\left(\hat{\beta}_{n}\right)}{Y_{0}\left(\hat{\beta}_{n}\right)-\hat{\beta}_{n} \hat{b}_{1} Y_{1}\left(\hat{\beta}_{n}\right)}\right]^{2} \\
& -\left(1+\hat{\beta}_{n}^{2} \hat{b}_{0}^{2}\right) \hat{R}_{0}^{2}\left[\frac{J_{1}\left(\hat{\beta}_{n} \hat{R}_{0}\right) Y_{0}\left(\hat{\beta}_{n} \hat{R}_{0}\right)-J_{0}\left(\hat{\beta}_{n} \hat{R}_{0}\right) Y_{1}\left(\hat{\beta}_{n} \hat{R}_{0}\right)}{Y_{0}\left(\hat{\beta}_{n} \hat{R}_{0}\right)+\hat{\beta}_{n} \hat{b}_{0} Y_{1}\left(\hat{\beta}_{n} \hat{R}_{0}\right)}\right]^{2} .
\end{aligned}
$$

The rate of flow through the annulus is

$$
\begin{aligned}
\hat{Q}(\hat{t})= & 2 \pi \int_{\hat{R}_{0}}^{1} \hat{r} \hat{u} \mathrm{~d} \hat{r} \\
= & \frac{\pi}{8}\left[-1+\hat{R}_{0}^{4}+\hat{c}_{1}\left(-1-2 \hat{R}_{0}^{2} \ln \hat{R}_{0}+\hat{R}_{0}^{2}\right)+2 \hat{c}_{2}\left(1-\hat{R}_{0}^{2}\right)\right] \\
& \quad-4 \pi \sum_{n=1}^{\infty} \frac{D_{n}}{\hat{\beta}_{n}^{4} E_{n}}\left[J_{1}\left(\hat{\beta}_{n}\right)-\hat{R}_{0} J_{1}\left(\hat{\beta}_{n} \hat{R}_{0}\right)\right. \\
& \left.\quad+\lambda_{n}\left(Y_{1}\left(\hat{\beta}_{n}\right)-\hat{R}_{0} Y_{1}\left(\hat{\beta}_{n} \hat{R}_{0}\right)\right)\right] e^{-\hat{\beta}_{n}^{2} \hat{t}} .
\end{aligned}
$$

In the case of zero slip at the walls, $\hat{b}_{0}=\hat{b}_{1}=0$, the characteristic equation reduces to

$$
J_{0}\left(\hat{\beta}_{n} \hat{R}_{0}\right) Y_{0}\left(\hat{\beta}_{n}\right)-J_{0}\left(\hat{\beta}_{n}\right) Y_{0}\left(\hat{\beta}_{n} \hat{R}_{0}\right)=0
$$

while the velocity and flow rate simplify to

$$
\begin{aligned}
& \hat{u}_{\text {no-slip }}=\frac{1}{4}\left[1-\hat{r}^{2}-\frac{\left(1-\hat{R}_{0}^{2}\right)}{\ln \hat{R}_{0}} \ln (\hat{r})\right]-2 \sum_{n=1}^{\infty} \frac{Z_{0}\left(\hat{\beta}_{n} \hat{r}\right) e^{-\hat{\beta}_{n}^{2} \hat{t}}}{\hat{\beta}_{n}^{3}\left[Z_{1}\left(\hat{\beta}_{n}\right)+\hat{R}_{0} Z_{1}\left(\hat{\beta}_{n} \hat{R}_{0}\right)\right]}, \\
& \hat{Q}_{\text {no-slip }}=\frac{\pi}{8}\left[1-\hat{R}_{0}^{4}+\frac{\left(1-\hat{R}_{0}^{2}\right)^{2}}{\ln \hat{R}_{0}}\right]-4 \pi \sum_{n=1}^{\infty} \frac{\left[Z_{1}\left(\hat{\beta}_{n}\right)-\hat{R}_{0} Z_{1}\left(\hat{\beta}_{n} \hat{R}_{0}\right)\right] e^{-\hat{\beta}_{n}^{2} \hat{t}}}{\hat{\beta}_{n}^{4}\left[Z_{1}\left(\hat{\beta}_{n}\right)+\hat{R}_{0} Z_{1}\left(\hat{\beta}_{n} \hat{R}_{0}\right)\right]},
\end{aligned}
$$

where

$$
Z_{k}\left(\hat{\beta}_{n} \hat{r}\right) \equiv J_{k}\left(\hat{\beta}_{n} \hat{r}\right)-J_{0}\left(\hat{\beta}_{n} \hat{R}_{0}\right) Y_{k}\left(\hat{\beta}_{n} \hat{r}\right) / Y_{0}\left(\hat{\beta}_{n} \hat{R}_{0}\right)
$$

This no-slip solution accords with the classical solution given by Müller [22].

For future reference, we provide in Table A5 the first five values of $\hat{\beta}_{n}$ for $\hat{R}_{0}=0.1,0.5$, and several values of $\hat{b}_{0}$ and $\hat{b}_{1}$. The leading eigenvalue $\hat{\beta}_{1}$ is shown in Fig. 17 as a function of the inner radius $\hat{R}_{0}$, for various values of $\hat{b}_{0}$ and $\hat{b}_{1}$. As expected, the eigenvalue increases as $\hat{R}_{0}$ increases, implying a faster decay of the start-up transient in a narrower annulus. 


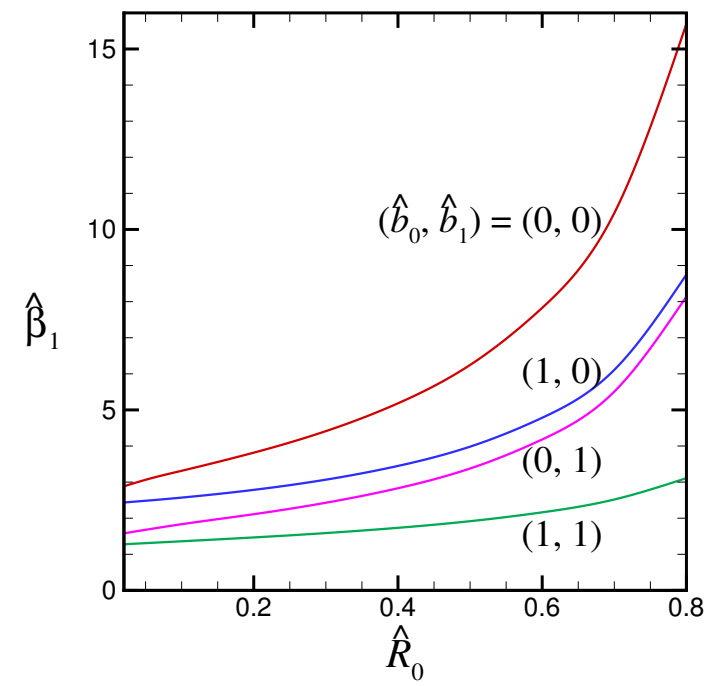

Figure 17: For starting Poiseuille flow through an annulus, the leading eigenvalue $\hat{\beta}_{1}$ as a function of the inner radius $\hat{R}_{0}$, for various values of the slip lengths $\hat{b}_{0}$ and $\hat{b}_{1}$.

When both inner and outer boundaries are non-slipping, $\hat{\beta}_{1}$ increases dramatically with $\hat{R}_{0}$, especially when $\hat{R}_{0}$ is close to unity. In sharp contrast, when both boundaries are slipping, $\hat{\beta}_{1}$ increases only mildly as $\hat{R}_{0}$ increases. We show for illustration in Fig. 18 some velocity profiles at various times for different values of the slip lengths. The effect of the boundary slip on the transient velocity profile is qualitatively the same as that already seen in Fig. 9 for flow through a parallel-plate channel.

\section{Concluding remarks}

We have presented a collection of problems and their analytical solutions for start-up internal flows subject to the Navier boundary slip condition. Although some of these problems have been studied previously, they are formulated here to cover more general cases (e.g., disparate slip lengths on different walls) than those in the existing literature. Most of the solutions presented in this paper are therefore reported for the first time. These analytical solutions are of archival value and may be used as benchmarks for numerical models for more complicated start-up slip flows. Further analytical studies may also be carried out on the basis of the 

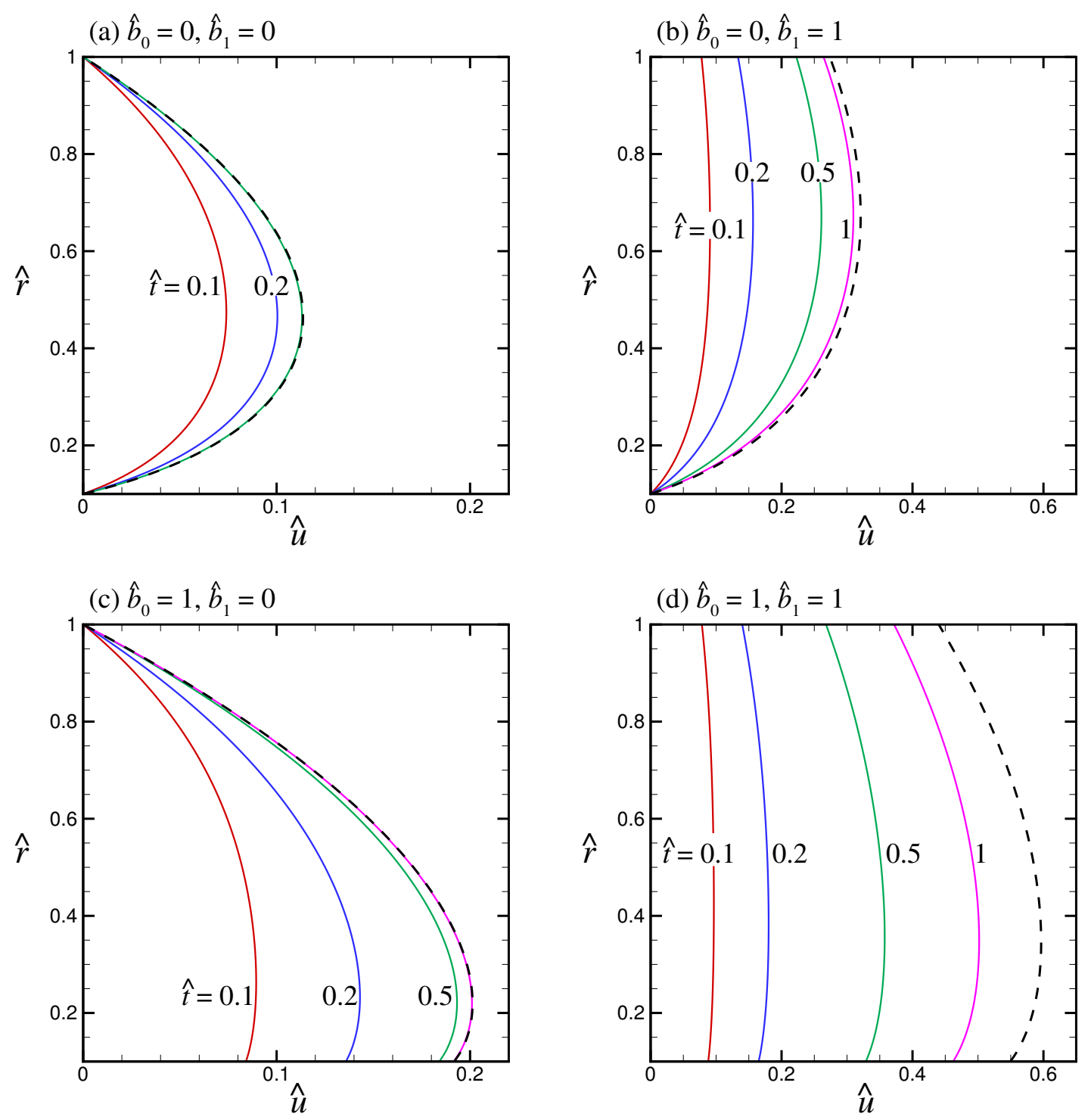

Figure 18: For starting Poiseuille flow through an annulus of inner radius $\hat{R}_{0}=0.1$, the velocity profile $\hat{u}(\hat{r})$ at various times, for (a) $\hat{b}_{0}=0, \hat{b}_{1}=0$; (b) $\hat{b}_{0}=0, \hat{b}_{1}=1$; (c) $\hat{b}_{0}=1$, $\hat{b}_{1}=0$; (d) $\hat{b}_{0}=1, \hat{b}_{1}=1$. The dashed lines denote the steady-state velocity profiles. 
present problems. We may, for example, follow Kaoullas and Georgiou $[14,23]$ to investigate start-up flow under the more advanced slip yield stress and slip relaxation effects.

\section{Acknowledgments}

The work was suggested to the author by Prof. C.Y. Wang of Michigan State University. Financial support was given by the Research Grants Council of the Hong Kong Special Administrative Region, China, through General Research Fund Project No. 17206615.

\section{Appendix}

Table A1: For starting Couette flow between two parallel flat plates, the first five eigenvalues $\hat{\beta}_{n}$ and Fourier coefficients $\hat{A}_{n}$, for some values of the slip lengths $\hat{b}_{0}, \hat{b}_{1}$. The eigenvalues $\hat{\beta}_{n}$ are also for starting Poiseuille flow through a parallel-plate channel.

\begin{tabular}{c|cc|cc|cc|cc}
\hline & \multicolumn{2}{|c|}{$\hat{b}_{0}=\hat{b}_{1}=0$} & \multicolumn{2}{c|}{$\hat{b}_{0}=0, \hat{b}_{1}=1$} & \multicolumn{2}{c|}{$\hat{b}_{0}=1, \hat{b}_{1}=0$} & \multicolumn{2}{c}{$\hat{b}_{0}=\hat{b}_{1}=1$} \\
\cline { 2 - 9 }$n$ & $\hat{\beta}_{n}$ & $\hat{A}_{n}$ & $\hat{\beta}_{n}$ & $\hat{A}_{n}$ & $\hat{\beta}_{n}$ & $\hat{A}_{n}$ & $\hat{\beta}_{n}$ & $\hat{A}_{n}$ \\
\hline 1 & 3.14159 & .63662 & 2.02876 & .82463 & 2.02876 & .16119 & 1.30654 & .32521 \\
2 & 6.28319 & .31831 & 4.91318 & .39150 & 4.91318 & .01557 & 3.67319 & .03301 \\
3 & 9.42478 & .21221 & 7.97867 & .24685 & 7.97867 & .00382 & 6.58462 & .00655 \\
4 & 12.56637 & .15915 & 11.08554 & .17897 & 11.08554 & .00144 & 9.63168 & .00217 \\
5 & 15.70796 & .12732 & 14.20744 & .14008 & 14.20744 & .00069 & 12.72324 & .00095 \\
\hline
\end{tabular}


Table A2: For starting Couette flow between two rotating coaxial cylinders, the first five eigenvalues $\hat{\beta}_{n}$ for some values of the inner radius $\hat{R}_{0}$ and the slip lengths $\hat{b}_{0}, \hat{b}_{1}$.

\begin{tabular}{c|cc|cc|cc|cc}
\hline & \multicolumn{2}{|c|}{$\hat{b}_{0}=\hat{b}_{1}=0$} & \multicolumn{2}{c|}{$\hat{b}_{0}=0, \hat{b}_{1}=1$} & \multicolumn{2}{c|}{$\hat{b}_{0}=1, \hat{b}_{1}=0$} & \multicolumn{2}{c}{$\hat{b}_{0}=\hat{b}_{1}=1$} \\
\cline { 2 - 9 }$\hat{R}_{0}$ & 0.1 & 0.5 & 0.1 & 0.5 & 0.1 & 0.5 & 0.1 & 0.5 \\
\hline$\hat{\beta}_{1}$ & 3.94094 & 6.39316 & 1.87926 & 2.97257 & 3.83899 & 4.76208 & 1.84315 & 2.08393 \\
$\hat{\beta}_{2}$ & 7.33057 & 12.62470 & 5.53222 & 9.39442 & 7.05042 & 10.25372 & 5.34841 & 7.27903 \\
$\hat{\beta}_{3}$ & 10.74838 & 18.88893 & 8.97468 & 15.69119 & 10.27261 & 16.24050 & 8.59711 & 13.14384 \\
$\hat{\beta}_{4}$ & 14.18864 & 25.16241 & 12.42190 & 21.97946 & 13.53341 & 22.37973 & 11.85353 & 19.24829 \\
$\hat{\beta}_{5}$ & 17.64331 & 31.43971 & 15.87978 & 28.26534 & 16.83667 & 28.57935 & 15.14507 & 25.43573 \\
\hline
\end{tabular}

Table A3: For starting Poiseuille flow through a rectangular channel, the first five eigenvalues $\hat{\gamma}_{m}$ for some values of the channel width $\hat{a}$ and the slip length $\hat{b}$. The other eigenvalue $\hat{\alpha}_{n}$ is equal to $\hat{\gamma}_{m}$ at $\hat{a}=1$.

\begin{tabular}{c|cc|cc|cc|cc}
\hline$\hat{a}$ & \multicolumn{2}{|c|}{0.5} & \multicolumn{2}{c|}{1} & \multicolumn{2}{c|}{2} & \multicolumn{2}{|c}{5} \\
\hline$\hat{b}$ & 0.5 & 1 & 0.5 & 1 & 0.5 & 1 & 0.5 & 1 \\
\hline$\hat{\gamma}_{1}$ & 1.72067 & 1.30654 & 1.07687 & 0.86033 & 0.63230 & 0.53844 & 0.28577 & 0.26277 \\
$\hat{\gamma}_{2}$ & 6.85124 & 6.58462 & 3.64360 & 3.42562 & 1.96758 & 1.82180 & 0.86116 & 0.80671 \\
$\hat{\gamma}_{3}$ & 12.87460 & 12.72324 & 6.57833 & 6.43730 & 3.40702 & 3.28917 & 1.44562 & 1.38192 \\
$\hat{\gamma}_{4}$ & 19.05867 & 18.95497 & 9.62956 & 9.52933 & 4.90594 & 4.81478 & 2.04005 & 1.97855 \\
$\hat{\gamma}_{5}$ & 25.29057 & 25.21203 & 12.72230 & 12.64529 & 6.43388 & 6.36115 & 2.64284 & 2.58704 \\
\hline
\end{tabular}


Table A4: For starting Poiseuille flow through a circular channel, the first five eigenvalues $\hat{\beta}_{n}$ for some values of the slip length $\hat{b}$.

\begin{tabular}{c|cccc}
\hline$\hat{b}$ & 0.1 & 0.5 & 1 & 2 \\
\hline$\hat{\beta}_{1}$ & 2.17950 & 1.59945 & 1.25578 & 0.94077 \\
$\hat{\beta}_{2}$ & 5.03321 & 4.29096 & 4.07948 & 3.95937 \\
$\hat{\beta}_{3}$ & 7.95688 & 7.28839 & 7.15580 & 7.08638 \\
$\hat{\beta}_{4}$ & 10.93633 & 10.36583 & 10.27099 & 10.22246 \\
$\hat{\beta}_{5}$ & 13.95803 & 13.47188 & 13.39840 & 13.36115 \\
\hline
\end{tabular}

Table A5: For starting Poiseuille flow through a concentric annulus, the first five eigenvalues $\hat{\beta}_{n}$ for some values of the inner radius $\hat{R}_{0}$ and the slip lengths $\hat{b}_{0}, \hat{b}_{1}$.

\begin{tabular}{c|cc|cc|cc|cc}
\hline \multirow{2}{*}{$\hat{R}_{0}$} & \multicolumn{2}{|c|}{$\hat{b}_{0}=\hat{b}_{1}=0$} & \multicolumn{2}{c|}{$\hat{b}_{0}=0, \hat{b}_{1}=1$} & \multicolumn{2}{c|}{$\hat{b}_{0}=1, \hat{b}_{1}=0$} & \multicolumn{2}{c}{$\hat{b}_{0}=\hat{b}_{1}=1$} \\
\cline { 2 - 9 } & 0.1 & 0.5 & 0.1 & 0.5 & 0.1 & 0.5 & 0.1 & 0.5 \\
\hline$\hat{\beta}_{1}$ & 3.31394 & 6.24606 & 1.83398 & 3.37698 & 2.57090 & 3.98268 & 1.36077 & 1.91724 \\
$\hat{\beta}_{2}$ & 6.85758 & 12.54687 & 5.19869 & 9.50466 & 5.83217 & 9.79906 & 4.30707 & 6.94481 \\
$\hat{\beta}_{3}$ & 10.37742 & 18.83642 & 8.68390 & 15.75580 & 9.18260 & 15.94118 & 7.57001 & 12.93000 \\
$\hat{\beta}_{4}$ & 13.88642 & 25.12285 & 12.17873 & 22.02529 & 12.58163 & 22.15961 & 10.92712 & 19.09710 \\
$\hat{\beta}_{5}$ & 17.38963 & 31.40800 & 15.67414 & 28.30088 & 16.00916 & 28.40598 & 14.33074 & 25.31983 \\
\hline
\end{tabular}




\section{References}

[1] Bestman AR, Ikonwa IO, Mbeledogu IU (1995) Transient slip flow. Int J Energy Res $19: 275-277$

[2] de Socio LM, Marino L (2002) Slip flow and temperature jump on the impulsively started plate. Int J Heat Mass Transfer 45:2169-2175

[3] Castellões FV, Cardoso CR, Couto P, Cotta RM (2007) Transient analysis of slip flow and heat transfer in microchannels. Heat Transfer Eng 28(6):549-558

[4] Wu YH, Wiwatanapataphee B, Hu M (2008) Pressure-driven transient flows of Newtonian fluids through microtubes with slip boundary. Phys A: Stat Mech Appl $387(24): 5979-5990$

[5] Wiwatanapataphee B, Wu YH, Hu M, Chayantrakom K (2009) A study of transient flows of Newtonian fluids through micro-annuals with a slip boundary. J Phys A: Math Theor 42(6):065206

[6] Wiwatanapataphee B, Wu YH, Suharsono S (2014) Transient flows of Newtonian fluid through a rectangular microchannel with slip boundary. Abstract and Applied Analysis, Article ID 530605

[7] Crane LJ, McVeigh AG (2015) Slip flow along an impulsively started cylinder. Arch Appl Mech 85:831-836

[8] Wang CY (2008) The starting flow in ducts filled with a Darcy-Brinkman medium. Transp Porous Med 75:55-62

[9] Wang CY (2011) Unsteady flow in a circular sector duct. Int J Nonlinear Mech 46:291295

[10] Wang CY (2012) Starting flow in a rotating parallel plate channel. Acta Mech Sinica 28(5):1271-1276 
[11] Avramenko AA, Tyrinov AI, Shevchuk IV (2015) An analytical and numerical study on the start-up flow of slightly rarefied gases in a parallel-plate channel and a pipe. Phys Fluids 27:042001

[12] Avramenko AA, Tyrinov AI, Shevchuk IV (2015) Start-up slip flow in a microchannel with a rectangular cross section. Theor Comput Fluid Dyn 29:351-371

[13] Matthews MT, Hastie KM (2012) An analytical and numerical study of unsteady channel flow with slip. ANZIAM J 53(4):321-336

[14] Kaoullas G, Georgiou GC (2015) Start-up and cessation Newtonian Poiseuille and Couette flows with dynamic wall slip. Meccanica 50:1747-1760

[15] Wang CY (2011) Two-fluid oscillatory flow in a channel. Theor Appl Mech Lett 1:032007

[16] Ng CO, Wang CY (2011) Oscillatory flow through a channel with stick-slip walls: Complex Navier's slip length. J Fluids Eng 133:014502

[17] Carslaw HS, Jaeger JC (1986) Conduction of heat in solids: second edition. Oxford University Press, Oxford. ISBN 0-19-853368-3

[18] Vinogradova OI (1995) Drainage of a thin liquid film confined between hydrophobic surfaces. Langmuir 11:2213-2220

[19] White FM (2006) Viscous fluid flow: third edition. McGraw-Hill, New York. ISBN 007-124493-X

[20] Leal LG (2007) Advanced transport phenomena. Cambridge University Press, Cambridge. ISBN 978-0-521-84910-4

[21] Szymanski F (1932) Quelques solutions exactes des équations de l'hydrodynamique de fluide visqueux dans le cas d'un tube cylindrique. J Math Pures Appl 11:67-107

[22] Müller W (1936) Zum problem der anlaufströmung einer flüssigkeit im geraden rohr mit Kreisring- und Kreisquerschnitt. ZAMM 16(4):227-238

[23] Kaoullas G, Georgiou GC (2013) Slip yield stress effects in start-up Newtonian Poiseuille flows. Rheol Acta 52:913-925 